\title{
1 Reward certainty and preference bias selectively shape voluntary decisions
}

2 Wojciech Zajkowski *, Dominik Krzemiński *, Jacopo Barone, Lisa Evans, Jiaxiang Zhang

3 Cardiff University Brain Research Imaging Centre, School of Psychology, Cardiff University,

4 Cardiff CF24 4HQ, United Kingdom

$5 \quad$ * The authors equal contributed equally to this work.

7 Correspondence should be addressed to:

8 Wojciech Zajkowski (zajkowskiw@ cardiff.ac.uk) or Jiaxiang Zhang (zhangj73@ cardiff.ac.uk)

\section{Conflict of Interest}

11 The authors declare no competing financial interests.

\section{Acknowledgements}

14 This study was supported by a European Research Council starting grant (716321). WZ was 15 supported by a $\mathrm{PhD}$ studentship from Cardiff University School of Psychology. DK was 16 supported by a PhD studentship from the Engineering and Physical Sciences Research Council 17 (1982622). We thank Sabina Baltruschat for assisting in data collection, and Craig Hedge and 18 Petroc Sumner for comments. 


\section{Abstract}

20 Choosing between equally valued options can be a conundrum, for which classical decision

21 theories predicted a prolonged response time (RT). Paradoxically, a rational decision-maker

22 would need no deliberative thinking in this scenario, as outcomes of alternatives are indifferent.

23 How individuals choose between equal options remain unclear. Here, we characterized the

24 neurocognitive processes underlying such voluntary decisions, by integrating advanced cognitive

25 modelling and EEG recording in a probabilistic reward task, in which human participants chose

26 between pairs of cues associated with identical reward probabilities at different levels. We

27 showed that higher reward certainty accelerated RT. At each certainty level, participants

28 preferred to choose one cue faster and more frequently over the other. The behavioral effects on

29 RT persisted in simple reactions to reward cues. By using hierarchical Bayesian parameter

30 estimation for an accumulator model, we showed that the certainty and preference effects were

31 independently associated with the rate of evidence accumulation during decisions, but not with

32 visual encoding or motor execution latencies. Time-resolved multivariate pattern classification of

33 EEG evoked response identified significant representations of reward certainty and preference

34 choices as early as $120 \mathrm{~ms}$ after stimulus onset, with spatial relevance patterns maximal in

35 middle central and parietal electrodes. Furthermore, EEG-informed computational modelling

36 showed that the rate of change between N100 and P300 event-related potentials reflected

37 changes in the model-derived rate of evidence accumulation on a trial-by-trial basis. Our findings

38 suggested that reward certainty and preference collectively shaped voluntary decisions between

39 equal options, providing a mechanism to prevent indecision or random behavior.

40 Keywords: decision making, reward certainty, preference bias, EEG, cognitive modelling 


\section{Introduction}

42 Cognitive flexibility enables decision strategies adaptive to environmental and motivational

43 needs (Schiebener and Brand, 2015). One characteristic of this ability is that harder decisions

44 often take longer. Evidence from neurophysiology (Gold and Shadlen, 2001), neuroimaging

45 (Heekeren et al., 2008) and modelling (Smith and Ratcliff, 2004) suggested an evidence

46 accumulation process for decision-making: information is accumulated over time, and a decision

47 is made when the accumulated evidence reached a threshold (Gold and Shadlen, 2007).

48 According to this framework, decision difficulty, and in turn response time (RT), is proportional

49 to the relative difference in the evidence supporting each option, consistent with results from

50 perceptual (Ditterich et al., 2003) and value-based (Oud et al., 2016) decisions.

51 What happens if decision difficulty reaches a tipping point with values of options being equal?

52 Classical stochastic decision models would predict a deadlock or indecision between equal

53 choices (Pais et al., 2013). In reality, individuals can choose between options with

54 indistinguishable certainty of receiving rewards (Voigt et al., 2019), such as deciding between

55 well-liked dishes in a good restaurant, or between underwhelming takeaways for a casual meal.

56 Paradoxically, economic analysis suggests that the closer options are in their reward probability,

57 the less beneficial to wait longer to decide. The benefit of "rushing to decisions" increases as

58 reward certainty gets larger because cognitive resources could be relocated elsewhere (Rustichini,

59 2009), predicting faster responses with higher reward certainty (Pirrone et al., 2018).

60 Furthermore, if choices are purely based on expected rewards, one may choose equal-valued

61 options randomly with the same probability. Nevertheless, choice behaviour deviates from

62 random in both lab-based (Zhang and Rowe, 2015) and consumer decisions (Wheeler, 1974), 
63 suggesting a possible bias between equal options, rendering some options more preferred than

64 others.

65 Previous research on equal choices raises three further unresolved issues. First, it is unclear how

66 reward certainty and preference bias impact on different sub-components of the decision-making

67 process. Second, functional imaging studies have localized the mesocorticolimbic dopaminergic

68 network to be involved in both reward certainty and preference processing (Tobler et al., 2007;

69 Abler et al., 2009). Less is known about how macroscopic brain activities relating to these effects

70 unfold in time. Third, conventional equal-choice paradigms commonly use subjective ratings to

71 quantify and equate values of options. This design has been shown to be vulnerable to value

72 fluctuations (Chen and Risen, 2010; Izuma and Murayama, 2013) that originate from voluntary

73 choices themselves (Festinger, 1957; Bem, 1967).

74 Here, we addressed these questions by combining advanced computational modelling and EEG

75 in a probabilistic reward task. Participants memorized six unambiguous cues associated with

76 three levels of reward certainty, and they made binary choices between cues with equal reward

77 certainty. Additional control conditions involved decisions between cues with unequal certainty

78 and unitary responses to single cues. This design enabled us to focus on the neurocognitive

79 processes of equal choices, while participants maintained a clear understanding of cue values for

80 rational decisions between unequal options.

81 We first examined how reward certainty influences behavior, and whether a preference bias was

82 present. We then fitted an accumulator model (Brown and Heathcote, 2008) to the behavioral

83 performance across reward certainty levels. Posterior model parameters were used to infer

84 whether the behavioral effects related to the decision process or visuomotor latencies unrelated

85 to decision-making. EEG data were analyzed with time-resolved multivariate pattern 
86 classification for decoding spatiotemporal representations of reward certainty and preference. To

87 establish a direct link between the decision process and its EEG signatures, we integrated

88 behavioral and EEG data into a joint hierarchical Bayesian model and tested the hypothesis that

89 electrophysiological activity reflects trial-by-trial changes in the speed of evidence accumulation

90 for decisions.

91 We demonstrated that reward certainty and spontaneous preference independently shape RT and

92 choices during equal choices. These behavioral effects extended further to other types of

93 decisions, affected the decision process and evoked distinct electrophysiological patterns. Our

94 findings highlighted that voluntary decisions are not random but subjected to exogenous and

95 endogenous control.

\section{Materials and Methods}

98 Participants

99 Twenty-three healthy participants were recruited from Cardiff University School of Psychology

100 participant panel (20 females; age range 19-32, mean age 22.7 years; 22 right-handed). All

101 participants had normal or corrected-to-normal vision, and none reported a history of

102 neurological or psychiatric illness. Written consent was obtained from all participants. The study

103 was approved by the Cardiff University School of Psychology Research Ethics Committee. 
106 The experiment was conducted in a dedicated EEG testing room. A computer was used to control

107 visual stimulus delivery and record behavioral responses. Visual stimuli were presented on a 24-

108 inch LED monitor (ASUS VG248) with a resolution of 1920 by 1080 pixels and a refresh rate of

$10960 \mathrm{~Hz}$, located approximately $100 \mathrm{~cm}$ in front of participants. Participants' responses were

110 collected from a response box (NATA technologies). The experiment was written in Matlab

111 (Mathworks; RRID: SCR_001622) and used the Psychophysics Toolbox Version 3 extensions

112 (Brainard, 1997; Kleiner et al., 2007, RRID: SCR_002881).

\section{Experimental design}

115 All participants performed a decision-making task with probabilistic rewards during EEG

116 recording (Fig. 1A). Before the decision task, the participants memorized 6 unambiguous cues in

117 different shapes and their associated probabilities of receiving a reward (Fig. 1B and see

118 Procedures). All the cues had the same color $(\mathrm{RGB}=246,242,92)$ on a black background $(100 \%$

119 contrast). Each cue was mapped onto one of the three reward certainty levels: high (a reward

120 probability of $100 \%$, i.e., always rewarded), medium (a reward probability of $80 \%$ ) and low (a

121 reward probability of $20 \%$ ), and hence there were two different cues associated with each reward

122 probability.

123 The participants were instructed to maximize the total accumulated reward in the decision-

124 making task. The task contained three conditions of trials: equal, unequal and forced. On an

125 equal trial, two different cues with the same reward certainty appeared on the left and right sides

126 of a central fixation point (e.g., $100 \%$ vs. $100 \%, 80 \%$ vs. $80 \%$ or $20 \%$ vs. $20 \%$ ). On an unequal

127 trial, two cues with different reward certainty levels appeared on both sides of the central fixation 
128 point (e.g., $100 \%$ vs. $20 \%, 100 \%$ vs. $80 \%$ or $80 \%$ vs. $20 \%$ ). On a forced trial, one of the six cues

129 appeared on either the left or right side of the fixation point. In equal and unequal trials,

130 participants chose the left or right cue via button presses with the right-hand index and middle

131 fingers. In forced trials, the participants responded to which side the single cue was presented

132 (i.e., left or right). In all trials, the reward was operationalized as 10 virtual "game points" that

133 did not have any tangible value. The probability of receiving the reward in a trial was either

$134100 \%, 80 \%$ or $20 \%$, which was determined by the chosen cue. It is worth noting that, in equal

135 trials, participants' decisions did not actually affect the probability of receiving the reward

136 because both options had equal reward certainty. In forced trials, if the participants chose the

137 wrong side with no cue presented ( $0.1 \%$ across all forced trials), no reward was given. Feedback

138 of rewarded (a "10 points" text message on the screen) or not rewarded (blank screen) was given

139 after each trial. The total game points awarded were presented at the bottom of the screen

140 throughout the experiment.

Procedure

143 Each experimental session comprised 640 trials, which were divided into 4 blocks of 160 trials.

144 Participants took short breaks between blocks and after every 40 trials within a block. The

145 mappings between the six reward cues and three levels of reward certainty were randomized

146 across participants. During breaks, the cues-reward mappings were explicitly presented on the

147 screen (Fig. 1B), and the participants could take as much time as they needed to memorize them.

148 After the first two blocks, all the cues were re-mapped to different reward probabilities. For

149 example, if a cue was associated with $100 \%$ reward probability in the first and second blocks, the 
150 same cue would be associated with either $80 \%$ or $20 \%$ reward probability in the third and fourth

151 blocks. The participants were encouraged to memorize the altered cue-probability associations

152 prior to the third block. This re-mapping procedure reduced the potential bias associated with

153 specific cue shapes.

154 Each block contained 64 equal trials (32 for $100 \%$ vs. 100\%, 16 for $80 \%$ vs. $80 \%$ and 16 for $20 \%$

155 vs. $20 \%$ ); 64 unequal trials (32 for $80 \%$ vs. $20 \%, 16$ for $100 \%$ vs. $80 \%$ and 16 for $100 \%$ vs. 20\%)

156 and 32 forced trials (16 for 100\%, 8 for $80 \%$ and 8 for 20\%) at a randomized order. This design

157 ensured the same number of trials with and without cues with the highest reward certainty

158 (100\%). Because there were two cues at each level of reward probability, different cue

159 combinations and their positions on the screen can give the same pair of reward probability (e.g.,

160 there are 8 possible combinations for $80 \%$ vs. $20 \%$ unequal trials). These different combinations

161 were counterbalanced across trials.

162 Each trial began with the presentation of a fixation point at the center of the screen, which was

163 presented for $500 \mathrm{~ms}$. After the fixation period, in the equal and unequal trials, two reward cues

164 appeared on the left and right sides of the screen with a horizontal distance of $4.34^{\circ}$ from the

165 fixation point. Both cues were vertically centered. In forced trials, only one reward cue appeared

166 on one side of the screen, and the side of cue appearance was randomized and counterbalanced

167 across trials. Cues were presented for a maximum of $2000 \mathrm{~ms}$, during which the participants

168 were instructed to make a left or right button press. The cues disappeared as soon as a response

169 was made, or the maximum duration was reached. The reaction time (RT) on each trial was

170 measured from the cue onset until participants made a response. Feedback (rewarded or not

171 rewarded) was given $200 \mathrm{~ms}$ after the reward cue offset and lasted $800 \mathrm{~ms}$, followed by a random

172 intertrial interval uniformly distributed between 1050 and $1150 \mathrm{~ms}$. As in our previous study 
173 (Zhang and Rowe, 2014), if the participant failed to respond within $2000 \mathrm{~ms}$ or responded within

$174100 \mathrm{~ms}$, no reward was given and a warning message "Too slow" or "Too fast" was presented for

$1751500 \mathrm{~ms}$.

177 Behavioral analysis

178 We excluded trials with RT faster than $200 \mathrm{~ms}$ (fast guesses). For each participant, trials with

179 RTs longer than 2.5 standard deviations from the mean RT were also excluded from subsequent

180 analysis. The discarded trials accounted for $1.5 \%$ of all trials.

181 We first analyzed the proportion of choices in equal trials to establish the existence of a

182 preference bias. In the equal condition, by definition, there was no "correct" or "incorrect"

183 response, because two cues in an equal trial had the same reward probability. For each pair of

184 cues with the same reward probability, we defined the preferred cue as the one chosen more

185 frequently than the other (non-preferred) one in equal trials. The categorization of preferred and

186 non-preferred cues was estimated separately between the first two and the last two blocks,

187 because of the cue-probability remapping after the first two blocks. At each level of reward

188 certainty, a preference bias was then quantified as the proportion of trials where the preferred

189 cue was chosen. The preference bias had a lower bound of 50\%, at which there was no choice

190 bias between the two cues with equal reward certainty.

191 In the unequal condition, we defined the decision accuracy as the proportion of choosing the cue

192 with higher reward probability, separately for each combination of reward probabilities (100\% vs.

$19380 \%, 100 \%$ vs. $20 \%$ and $80 \%$ vs. $20 \%$ ). Two-tailed one-sample t-tests compared the decision 
194 accuracy in the unequal condition against a chance level of 50\%, which would indicate non-

195 rational decisions (i.e., both high and low reward cues were chosen in $50 \%$ of trials).

196 To determine how reward certainty, preferences and other experimental factors influence RT, we 197 analyzed single-trial RT data with linear mixed-effects models (LMMs) using the lme4 package

198 (Bates et al., 2015b) in R (RRID: SCR_001905). The LMM is a hierarchical regression method

199 that distinguishes between fixed and random effects (Gueorguieva and Krystal, 2004). LMMs

200 take into account all single-trial data without averaging across trials and offer better control of

201 type 1 and type 2 errors than ANOVA (Baayen et al., 2008). Therefore, statistical inferences

202 from LMMs are robust to experimental designs with unbalanced trials across conditions

203 (Bagiella et al., 2000), which is an important feature suitable for the current study.

204 We designed two LMMs with different dependent variables and factors (Table 1). Model 1

205 analyzed the RTs from equal and forced trials, including choice type (equal or forced) and

206 reward certainty (high, medium or low) as factors. For the unequal condition, because each trial

207 had two cues with different levels of reward certainty that cannot be directly compared with

208 equal or forced trials, the RTs of unequal trials were analyzed separately in Model 2. The sum

209 and the absolute difference of the two reward probabilities in each unequal trial were included as

210 factors of RT, as they have been shown to affect choice behavior (Thaler, 1981; Teodorescu et

211 al., 2016; Ballard et al., 2017).

212 In all the LMMs, fixed effects structures included hypothesis-driven, design-relevant factors and

213 their interactions, and individual participants were included as the source of random variance

214 (random effect). We used a standard data-driven approach to identify the random effects

215 structure justified by the experimental design, which resulted in good generalization performance

216 (Barr et al., 2013; bust see Bates et al., 2015 for alternative methods). This approach starts with 
217 the maximal random effects structure (i.e., including all random slopes, intercepts and

218 interactions) and systematically simplified it until the LMM reaches convergence. Table 1 lists

219 the simplified random effects structures. The correlation structures of each fitted LMM was

220 assessed to avoid overfitting (Matuschek et al., 2017).

222 A cognitive model of voluntary decision-making

223 We further analyzed the behavioral data using a cognitive model, the Linear Ballistic

224 Accumulator (LBA) model (Brown and Heathcote, 2008). The LBA model is a simplified

225 implementation of a large family of sequential sampling models of decision-making (Ratcliff and

226 Smith, 2004; Bogacz et al., 2006; Gold and Shadlen, 2007; Zhang, 2012). It has been used to

227 examine cognitive processes during perceptual decision (Ho et al., 2009; Forstmann et al., 2010a)

228 voluntary choice (Zhang et al., 2012) and action initiation (Karahan et al., 2019).

229 Our model-based analysis served three purposes in the current study. First, we fitted a family of

230 LBA models with various model complexity to behavioral data of individual participants in

231 equal trials. By identifying the best-fitted model, we inferred how reward certainty and

232 preference modulated subcomponents of the evidence accumulation process during decision-

233 making. Second, we simulated the best fitted LBA model and examined whether model

234 simulations were consistent with the experimental data in forced and unequal conditions. This is

235 a stringent test of model generalizability because the experimental data in forced and unequal

236 trials were unseen by the model fitting procedure. Third, we linked the cognitive processes

237 identified by the LBA model to brain activities. This was achieved by incorporating a trial-by- 
238 trial measure of EEG activity regressors into the best-fitted model (Cavanagh et al., 2011; Nunez

239 et al., 2017, 2018, and see EEG-informed cognitive modelling).

240 In equal trials, the participants made binary choices between two cues associated with the same

241 reward certainty. The LBA model assumed that the decision of when and which to choose is

242 governed by a "horse race" competition between two accumulators $i \in\{1,2\}$ that accumulate

243 evidence over time supporting the two choice options (Fig. 1C). One accumulator is in favor of

244 the preferred cue, and the other accumulator is in favor of the non-preferred cue. The activations

245 of the accumulators represent the accumulated evidence. At the beginning of each trial, the initial

246 activations of the two accumulators are independently drawn from a uniform distribution

247 between 0 and $A$. The activation of each accumulator then increases linearly over time, and the

248 speed of accumulation (i.e., accumulation rate) varies as a Gaussian random variable with mean

$249 v_{i}$ and standard deviation $S_{i}$ across trials. The accumulation process terminates when the

250 activation of any accumulator reaches a response threshold $B(B>A)$ and the choice

251 corresponding to the winning accumulator is selected. The model prediction of RT is the sum of

252 the duration of the accumulation process and a constant non-decision time $T_{\text {er }}$, with the latter

253 accounts for the latency associated with other processes including stimulus encoding and action

254 execution (Brown and Heathcote, 2008; Nunez et al., 2018; Karahan et al., 2019).

256 Model parameter estimation and model selection

257 The LBA model has five key parameters: the mean $v$ and standard deviation $S$ of the 258 accumulation rate across trials, the decision threshold $B$, the standing point variability $A$ and the 259 non-decision time $T_{\mathrm{er}}$. To accommodate the empirical data, one or more model parameters need 
to vary between conditions. We evaluated a total of 21 variants of the LBA model with different

261 parameter constraints (Fig. 3A). First, the accumulation process may differ between the preferred

262 and non-preferred options, leading to $v$ or $S$ to vary between accumulators (preferred, non-

263 preferred). Second, reward certainty could modulate the accumulation process or visuomotor

264 latencies unrelated to decisions, leading to $v, S$ or $T_{\text {er }}$ to vary between three levels of reward

265 certainty $(100 \%, 80 \%$ and $20 \%)$. Third, the decision threshold $B$ was fixed between conditions,

266 because the trial order was randomized, and we do not expect the participants to systematically

267 vary their decision threshold before knowing the cues to be presented (Ratcliff and Smith, 2004).

268 During model-fitting, the decision threshold was fixed at 3 as the scaling parameter (Brown and

269 Heathcote, 2008), and all the other parameters allowed to vary between participants. Finally,

270 because the participants showed behavioral differences between reward certainty levels and

271 between preferred/non-preferred choices, we only estimated realistic models: those with at least

272 one parameter varied between reward certainty levels $\left(v, S\right.$ or $\left.T_{\mathrm{er}}\right)$ and at least one parameter

273 varied between accumulators $(v$ or $S)$.

274 We used a hierarchical Bayesian model estimation procedure to fit each LBA model variant to 275 individual participant's choices (the proportion of preferred and non-preferred choices) and RT

276 distributions in equal trials. The hierarchical model assumes that model parameters at the

277 individual-participant level are random samples drawn from group-level parameter distributions.

278 Given the observed data, Bayesian model estimation uses Markov chain Monte Carlo (MCMC)

279 methods to simultaneously estimate posterior parameter distributions at both the group level and

280 the individual-participant level. The hierarchical Bayesian approach has been shown to be more

281 robust in recovering model parameters than conventional maximum likelihood estimation

282 (Jahfari et al., 2013; Zhang et al., 2016). 
283 For group-level parameters ( $v, S, A$ and $T_{\text {er }}$ ), similar to previous studies (Annis et al., 2017), we

284 used weakly informed priors for their means $E($.$) and standard deviations \operatorname{std}($.$) :$

$$
\begin{aligned}
& E(v) \sim \mathcal{H}(2.5,1), \quad \operatorname{std}(v)=g(1,1), \\
& E(S) \sim \mathcal{H}(1,0.75), \quad s t d(S)=\mathscr{g}(1,1), \\
& E(A) \sim \mathcal{H}(2.5,1), \quad \operatorname{std}(A)=g(1,1), \\
& E\left(T_{\text {er }}\right) \sim \mathcal{H}(0.5,0.2), \quad s t d\left(T_{\text {er }}\right)=g(1,1),
\end{aligned}
$$

285 where $\mathcal{H}$ represents a positive normal distribution (i.e., truncated at 0 ) with parameterized mean 286 and standard deviation, and $g$ represents a Gamma distribution with parameterized mean and 287 standard deviation.

288 We used the hBayesDM package (Ahn et al., 2017) in $R$ for the hierarchal implementation of the 289 LBA model. For each of the 21 model variants, we generated four independent chains of 7,500 290 samples from the joint posterior distribution of the model parameters using Hamiltonian Monte 291 Carlo (HMC) sampling in Stan (Carpenter et al., 2017). HMC is an efficient method suitable for 292 exploring high-dimensional joint probability distributions (Betancourt, 2017). The initial 2,500 293 samples were discarded as burn-in. To assess the convergence of the Markov chains, we 294 calculated Gelman-Rubin convergence diagnostic $\hat{R}$ of each model (Gelman and Rubin, 1992) 295 and used $\hat{R}<1.1$ as a stringent criterion of convergence (Annis et al., 2017). We compared the 296 fitted LBA model variants using Bayesian leave-one-out information criterion (LOOIC). LOOIC 297 evaluates the model fit while considering model complexity, with lower values of LOOIC 298 indicating better out-of-sample model prediction performance (Vehtari et al., 2017). 
301 We used Bayesian inference to analyze the posterior distributions of group-level model

302 parameters (Berger and Bayarri, 2004). To evaluate if a parameter varies substantially between

303 two conditions, we calculated the proportion of posterior samples in which the parameter value

304 for one condition was greater than the other. To test if a parameter differs from a threshold value,

305 we calculated the proportion of the posteriors greater or smaller than the threshold. To avoid

306 confusion, we used $p$ to refer to classical frequentist $p$-values, and $P_{p \mid \mathrm{D}}$ to refer to Bayesian

307 inference results based on the proportion of posteriors supporting the testing hypothesis, given

308 the observed data.

Model simulations

311 To evaluate the model fit to the empirical data of equal choices, we calculated the posterior

312 prediction of the best fitted LBA model by averaging 100 iterations of model simulation using

313 posterior parameter estimates. Averaging across multiple iterations reduces potential biases when

314 sampling from posterior parameter estimates. Each of the 100 iterations generated simulated

315 behavioral responses (i.e., RTs and choices) of individual participants, with the same number of

316 trials per condition as in the actual experiment.

317 We further used the best fitted LBA model to simulate unequal and forced choice responses. This

318 allowed us to evaluate whether the model fitted to the equal choice data could characterize

319 behavioral patterns in other types of choices. For unequal choices, two accumulators representing

320 two cues with different reward certainty levels compete to reach the decision threshold, with

321 their parameters set to the posterior estimates from the fitted LBA model. For forced choices, a

322 single accumulator was set to reach to the decision threshold. Similar to the simulation of equal 
323 choices, for each participant, we averaged the predicted behavioral responses of unequal and

324 forced choices from 100 iterations of simulation. Each iteration contained the same number of

325 trials as in the experiment.

327 EEG data acquisition $\square$ and processing

328 EEG data were collected using a 32-channel Biosemi ActiveTwo device (BioSemi, Amsterdam).

329 Due to technical issues, EEG data collection was not successful in two participants, and therefore

330 all EEG data analyses were performed on the remaining 21 participants. EEG electrodes were

331 positioned at standard scalp locations from the International 10-20 system. Vertical and

332 horizontal eye movements were recorded using bipolar electrooculogram (EOG) electrodes

333 above and below the left eye as well as from the outer canthi. Additional electrodes were placed

334 on the mastoid processes. EEG recordings (range DC-419 Hz; sampling rate $2048 \mathrm{~Hz}$ ) were

335 referenced to linked electrodes located midway between POz and PO3/PO4 respectively and re-

336 referenced off-line to linked mastoids.

337 EEG data were pre-processed using EEGLab toolbox 13.4.4b (Delorme and Makeig, 2004,

338 RRID: SCR_007292) in Matlab. The raw EEG data were high-pass filtered at 0.1 Hz, low-pass

339 filtered at $100 \mathrm{~Hz}$ using Butterworth filters and downsampled to $250 \mathrm{~Hz}$. An additional $50 \mathrm{~Hz}$

340 notch filter was used to remove mains interference. We applied Independent Component

341 Analysis (ICA) to decompose continuous EEG data into 50 spatial components, using runica

342 function from the EEGLab toolbox. Independent components reflecting eye movement artifacts

343 were identified by the linear correlation coefficients between the time courses of independent

344 components and vertical and horizontal EOG recordings. Additional noise components were 
345 identified by visual inspection of the components' activities and scalp topographies. Artefactual

346 components were discarded, and the remaining components were projected back to the data

347 space.

348 After artifact rejection using ICA, the EEG data were low-pass filtered at $40 \mathrm{~Hz}$ and epoched

349 from -400 ms to $1000 \mathrm{~ms}$, time-locked to the onset of the stimulus (i.e., reward cues) in each trial.

350 Every epoch was baseline corrected by subtracting the mean signal from $-100 \mathrm{~ms}$ to $0 \mathrm{~ms}$ relative

351 to the onset of reward cues.

353 Event $\square$ related potential (ERP) analysis

354 We examined univariate differences in evoked responses between conditions in single EEG

355 electrodes. For each participant, trial-averaged ERPs were calculated from epochs of equal or

356 forced choices. For both equal and forced conditions, we tested for differences in ERPs between

357 three levels of reward certainty using a one-way repeated-measures ANOVA. Furthermore, we

358 tested for differences in ERPs between preferred and non-preferred choices in equal trials, using

359 a paired t-test. We performed statistical tests in all electrodes and all time points. Cluster-based

360 permutation tests (2000 iterations with maximum statistics) were used to correct for multiple

361 comparisons across electrodes and time points (Maris and Oostenveld, 2007).

\section{Multivariate pattern analysis}

364 We used time-resolved MVPA on pre-processed, stimulus-locked EEG data to assess reward-

365 specific and preference-specific information throughout the time course of a trial. In contrast to 
univariate ERP analysis, MVPA combines information represented across multiple electrodes, which has been shown to be sensitive in decoding information representation from multi-channel human electrophysiological data (Cichy et al., 2014; Dima et al., 2018).

We conducted three MVPA analysis to identify the latency and spatial distribution of the EEG multivariate information. The first was to decode reward certainty levels in equal choices (e.g., equal trials with two $100 \%$ reward cues versus equal trials with two $80 \%$ cues). The second was to decode preferred versus non-preferred choices in equal trials. The third was to decode between equal and forced choices with the same reward certainty (e.g., equal trials with two $100 \%$ cues versus forced trials with a $100 \%$ cue).

375 Each analysis was formed as one or multiple binary classification problems, and the data feature 376 for classification included EEG recordings from all 32 electrodes. In each analysis, at each 377 sampled time point (-400 ms to $1000 \mathrm{~ms}$ ) and for each participant, we trained linear support 378 vector machines (SVM) (Garrett et al., 2003) using the 32-channel EEG data and calculated the mean classification accuracy following a stratified ten-fold cross-validation procedure. In all

380 MVPA, we included the EEG data from $400 \mathrm{~ms}$ before cue onset as a sanity check, because one 381 would not expect significant classification before the onset of reward cues.

382 In each cross-validation, $90 \%$ of the data was used as a training set, and the remaining $10 \%$ of 383 the data was used as a test set. In some analysis (e.g., equal trials with $100 \%$ cues versus equal 384 trials with $80 \%$ cues), the number of samples belonging to the two classes was unbalanced in the 385 training set. We used a data-driven over-sampling approach to generate synthetic instances for 386 the minor class until the two classes had balanced samples (Zhang and Wang, 2011). The 387 synthetic instances were generated from Gaussian distributions with the same mean and variance 388 as in the original minority class data. Training set data were standardized with z-score 
normalization to have a standard normal distribution for each feature. The normalization parameters estimated from the training set was then applied separately to the test set to avoid

391 overfitting. To reduce data dimensionality, we performed principal component analysis to the

392 training set data and selected the number of components that explained over $99 \%$ of the variance

393 in the training set. The test set data were projected to the same space with reduced dimensions by

394 applying the eigenvectors of the chosen principal components. We then trained SVM to

395 distinguish between the two classes (i.e., conditions) and evaluate the classification accuracy

396 using the test set data. The procedure was repeated ten times with different training and test sets,

397 and the classification accuracies were averaged from the ten-fold cross-validation. We used the

398 SVM implementation in MATLAB Machine Learning and Statistics Toolbox. The trade-off

399 between errors of the SVM on training data and margin maximization was set to 1.

400 To estimate the significance of the classification performance, we used two-tailed one-sample t-

401 test to compare classification accuracies across participants against the $50 \%$ chance level. To

402 account for the number of statistical tests at multiple time points, we used cluster-based

403 permutation (Maris and Oostenveld, 2007) to control the family-wise error rate at the cluster 404 level from 2000 permutations.

407 To evaluate the relative importance of each feature (i.e., EEG electrode) to the classification 408 performance, we calculated the weight vector of SVMs. For each classification problem, we 409 retrained the SVM at each time point with all the data included in the training set and obtained 410 the SVM weight vector. The weight vectors were then transformed into interpretable spatial 
411 patterns by multiplying the data covariance matrix (Haufe et al., 2014). The group spatial

412 patterns were calculated by averaging across participants and from all time points which had

413 significant classification accuracy.

415 Estimation of single-trial ERP components

416 We estimated two ERP components from single-trial EEG data in equal trials: N100 and P300,

417 which were subsequently used to inform cognitive modelling. The visual N100 is related to

418 visual processing (Mangun and Hillyard, 1991) and the P300 is related to evidence accumulation

419 during decision making (Kelly and O’Connell, 2013; Twomey et al., 2015).

420 To improve the signal-to-noise ratio of single-trial ERP estimates, we used a procedure similar to

421 previous studies (Kayser and Tenke, 2003; Parra et al., 2005; Nunez et al., 2018). For each

422 participant, we first performed singular value decomposition (SVD) to the grand averaged ERP

423 data across all equal trials. The first SVD component is the one that explained the most variance

424 in the averaged ERP waveforms, and its weight vector provides an optimal spatial filter to detect

425 the ERP waveforms. Next, we applied the optimal spatial filter as a channel weighting function

426 to single-trial EEG data.

427 The single-trial EEG data filtered with the SVD-based weighting function was then used to

428 identify the peak-latency and peak-amplitude of the N100 and P300 components. For N100, we

429 searched for the peak negative amplitude in a window centered at the group-level N100 latency

$430(112 \mathrm{~ms})$ and started at $60 \mathrm{~ms}$. The lower bound of the search window was determined by the

431 evidence that the visual onset latency is $\sim 60 \mathrm{~ms}$ in V1 (Schmolesky et al., 2017). For P300, we

432 searched for a peak positive amplitude in a window centered at the group-level P300 latency 
433 (324 ms). For both N100 and P300, the search window had a length of $104 \mathrm{ms,} \mathrm{similar} \mathrm{to} \mathrm{a}$

434 previous study (Nunez et al., 2018).

436 EEG-informed cognitive modeling

437 From the behavioral responses of each participant and each condition, we used the LBA model to

438 estimate a fixed mean accumulation rate across trials (see Model parameter estimation and

439 model selection). Recent studies showed that the variability of the P300 component closely

440 relates to the rate of evidence accumulation during decision making (Twomey et al., 2015). We

441 therefore extended the best fitted LBA model with EEG-informed, single-trial regressors, which

442 estimated the effect of trial-by-trial variability in EEG activity on the mean accumulation rate

443 (Hawkins et al., 2015; Nunez et al., 2017).

444 The main regressor of interest was the slope of change between the P300 and N100 components,

445 which were estimated as the ratio of the P300-N100 peak-amplitude difference and the P300-

446 N100 peak-latency difference in each equal trial. We also tested four additional regressors from

447 individual ERP components: P300 amplitude, P300 latency, N100 amplitude and N100 latency.

448 All the EEG regressors were obtained from the estimations of single-trial ERP components in

449 equal choice trials. To obtain a meaningful intercept, the regressors were mean-centered and

450 rescaled to have a unit standard deviation.

451 Each EEG regressor was tested in a linear regression model, using the same Bayesian

452 hierarchical model estimation procedure as in the behavioral modelling analyses. For each 453 regression model, we assumed that the mean accumulation rates of both accumulators $v_{1}(t)$ and 
$454 v_{2}(t)$ (i.e., the one in favor of the preferred option and the other one in favor of the non-preferred

455 option) are influenced by the EEG regressor of interest on a trial-by-trial basis:

$$
v_{1}(t)=\overline{v_{1}}+\beta \cdot E E G(t), v_{2}(t)=\overline{v_{2}}+\beta \cdot E E G(t)
$$

457 while $t=1,2,3, \ldots$ represents the equal choice trials, and $\overline{v_{1}}$ and $\overline{v_{2}}$ are the intercepts. The

458 regression coefficient $\beta$ represents the effect of EEG regressor on the mean accumulation rates.

Software and data accessibility

461 The scripts for behavioral modelling and EEG analysis are open-source and freely available

462 online (https://github.com/ccbrain/voluntary-decision-eeg). We have also made the behavioral

463 and EEG data open access (https://doi.org/10.6084/m9.figshare.9989552.v1).

\section{Results}

466 We examined the effects of reward certainty and spontaneous preference on behavior and EEG

467 activity during voluntary decisions. In a probabilistic reward task, participants chose between

468 two options with the same reward certainty (equal trials) at high (100\%), medium (80\%) or low

469 (20\%) levels. In additional control conditions, the participants made binary choices between

470 options with different levels of reward certainty (unequal trials) or responded to the location of a

471 single reward cue (forced trials). Below, we first reported behavioral results. We then fitted LBA

472 models (Brown and Heathcote, 2008) to the choices and RT distributions of equal trials and

473 make inferences about the model parameters that quantify an evidence accumulation process

474 during voluntary decisions. Next, we performed univariate and multivariate analyses of EEG 
475 data to identify spatiotemporal representations of reward certainty and preference information as

476 well as their time courses. We then extended the best-fitted LBA model with single-trial

477 measures of EEG activity to test whether trial-to-trial variations in EEG data relates to the rate of

478 evidence accumulation across trials.

481 In equal choice trials, we calculated the preference bias as the proportion of choosing the cue

482 with higher frequency in equal trials (i.e., the preferred cue). At each level of reward certainty,

483 there was a strong preference bias (>50\%) for choosing one reward cue over the other (Fig. 2A;

484 high: 95\% CI [0.682, 0.765]; medium: 95\% CI [0.679, 0.759]; low: 95\% CI [0.669, 0.745]). A

485 repeated-measures ANOVA showed no significant difference in preference bias between reward

486 certainty levels $(F(2,44)=0.2, p=0.81)$. Therefore, although the two options had the same

487 reward certainty, participants did not make their choices randomly. Because the cue-probability

488 mapping was randomized across participants and re-mapped within each session, the observed

489 preference bias could not be readily explained by a group-level preference towards any specific

490 cue, but rather a spontaneous bias at the individual level.

491 In unequal choice trials, as expected, the cues with higher reward certainty levels were chosen

492 more often, as evidenced by the above-chance decision accuracies in all conditions (Fig. 2A;

493 high vs. medium: $t(22)=16.08,95 \%$ CI $[0.774,1], p<0.001$; high vs. low: $t(22)=23.31,95 \%$

494 CI $[0.862,1], p<0.001$; medium vs. low: $t(22)=20.97,95 \%$ CI $[0.834,1], p<0.001$; one-

495 sample t-test against the 0.5 chance level). A repeated-measures ANOVA showed significant

496 differences in decision accuracy between reward certainty levels $(F(2,44)=28.17, p<0.001)$. 
Post-hoc pairwise comparison with Tukey's correction indicated that accuracy in the high vs.

498 low certainty condition $(93.8 \%)$ was significantly higher than in the high vs. medium $(84.3 \%$;

$499 t(44)=5.267, p<0.001)$ and the medium vs. low $(80.7 \% ; t(44)=7.265, p<0.001)$ conditions.

500 These results suggested that the participants remembered the cue-reward mapping for rational

501 choice behavior.

\section{Behavioral results: response time}

504 We used a linear mixed-effects model (LMM) to quantify the influence of experimental factors

505 on RTs in equal and forced choices (Fig. 2B, Model 1 in Table 1). The fixed effects included

506 reward certainty, choice type (equal vs. forced), preference (choosing the preferred vs. the non-

507 preferred option), cue re-mapping (before vs. after the cue-reward remapping halfway through

508 each session) and their meaningful interactions (Figs. 2D-2F). The LMM included individual

509 participants as random intercepts and data-driven random effects structures (Barr et al., 2013).

510 The participants were faster in responding with the preferred than the non-preferred option (Fig.

$5112 \mathrm{D}, \beta=-0.063,95 \% \mathrm{CI}[-0.027,-0.991], p<0.001)$ and RTs decreased as the reward certainty

512 increased $(\beta=-0.101,95 \%$ CI $[-0.067,-0.135], p<0.001)$. The $\mathrm{RT}$ in equal choice trials were

513 longer than that in forced trials $(\beta=-0.292,95 \%$ CI $[-0.201,-0.384], p<0.001)$. The effect of

514 reward certainty on RT was stronger in equal choices than that in forced choices, supported by a

515 significant interaction between the two main effects $(\beta=0.045,95 \%$ CI $[0.025,0.066], p<$

$5160.001)$.

517 Participants had slower responses after memorizing a new set of cue-reward associations, 518 indicated by a significant main effect in RT before and after cue re-mapping $(\beta=0.149,95 \%$ CI 
$519[0.096,0.201], p<0.001)$. The significant interaction between cue re-mapping and reward

520 certainty suggested that the increase in RT was more pronounced in trials with lower reward

521 certainty (Fig. 2F, $\beta=-0.039,95 \%$ CI $[-0.051,-0.026], p<0.001$ ). The interaction between cue

522 re-mapping and choice type (Fig. $2 \mathrm{G}, \beta=-0.247,95 \%$ CI $[-0.192,-0.302], p<0.001$ ) further

523 indicated that this pattern was mainly associated with equal choices than forced responses.

524 Because evaluating the reward certainty of a cue was likely associated with additional cognitive

525 load after cue re-mapping, the observed RT difference before and after cue re-mapping implies

526 that, in equal choices, the participants evaluated both cues throughout the experimental session.

527 In a second LMM, we analyzed RTs in unequal trials (Model 2 in Table 1), including the sum 528 and difference of the reward certainty of two cues in each trial as fixed effects. The sum of the

529 two reward probabilities in unequal trials was negatively associated with RT (Fig. 2E, $\beta=-0.071$,

$53095 \%$ CI $[-0.032,-0.110], p<0.001)$, consistent with previous studies that the total reward

531 magnitude influences decision-making (Pirrone et al., 2014; Teodorescu et al., 2016).

\section{Cognitive modelling of behavioral data}

534 We showed that the behavioral performance in equal choices was modulated by both reward

535 certainty and spontaneous preference. Similar to previous studies (Forstmann et al., 2010b;

536 Mulder et al., 2010, 2014; Zhang et al., 2012), we conceptualized that a decision between two

537 equal options is described by an LBA model. The model assumes that the momentary evidence

538 for choosing the preferred and non-preferred options accumulate independently over time, until a

539 decision threshold is reached (Fig. 1C). 
540 To identify the cognitive processes that led to the observed behavioral differences, we compared

54121 variants of the LBA model. The model variants differed systematically in their constraints on

542 whether the rate of evidence accumulation rate and the non-decision time could change between

543 reward certainty levels or between preferred/non-preferred options. For each model variant, we

544 used hierarchal Bayesian modelling with MCMC parameter estimation routine to estimate the

545 posterior distributions of the model parameters, given the observed choice and RT distribution

546 from individual participants (see Model parameter estimation and model selection). To identify

547 the model with the best fit, we calculated the Bayesian leave-one-out information criterion

548 (LOOIC) score of each model (Vehtari et al., 2017).

549 Posterior parameter estimates in all the 21 model variants were converged after sufficient

550 samples (Gelman-Rubin convergence diagnostic $\hat{R} \leq 1.02$ for all parameters in all models). The

551 LOOIC scores suggested that the models with the mean accumulation rate varying between

552 reward certainty levels and between preference levels fitted the data better than others model

553 variants. The best-fitting model (i.e., the one with the lowest LOOIC score, Fig. 3A) had a fixed

554 non-decision time per participant with the standard deviation of the accumulation rate varying

555 between reward certainty levels and preferred/non-preferred options. To evaluate the model fit,

556 we simulated the best fitting model and compared its posterior predictions with the observed

557 equal choice data (Fig. 3B). There was a good agreement between the observed data and the

558 model simulations across reward certainty levels and choice preferences.

559 For the best fitting model (Fig. 4A), we compared the posterior estimates of the group-level

560 parameters between conditions (Fig. 4B and Fig. 4C). There was strong evidence for a larger

561 reward certainty being associated with higher mean $\left(v\right.$, high $>$ low: $P_{p \mid \mathrm{D}}=1$; high $>$ medium: $P_{p \mid \mathrm{D}}$

$562=0.999 ;$ medium $>$ low: $\left.P_{p \mid \mathrm{D}}=0.839\right)$ and standard deviation $\left(S\right.$, high $>$ low: $P_{p \mid \mathrm{D}}=1$; high $>$ 
563 medium: $P_{p \mid \mathrm{D}}=0.954$; medium $>$ low: $\left.P_{p \mid \mathrm{D}}=0.877\right)$ of the accumulation rate. There was also

564 strong evidence for a higher mean accumulation rate for the preferred than the non-preferred

565 options $\left(P_{p \mid \mathrm{D}}=0.999\right)$, and no evidence for a difference in the standard deviation of the

566 accumulation rate between preference levels $\left(P_{p \mid \mathrm{D}}=0.532\right)$. These results supported that

567 preferred cues and the ones with higher reward certainty were recalled and processed faster than

568 non-preferred cues. The cues with higher reward certainty were also associated with more

569 variable accumulation rate. Model comparisons further suggested that the latencies of early

570 visual encoding and motor execution were not influenced by reward certainty nor preference,

571 because the models with varying non-decision time had inferior fits.

572 Next, we evaluated whether the best fitting model could reproduce qualitative RT patterns in the

573 forced and unequal choices (Fig. 3C), which were unseen by the parameter estimation procedure

574 (see Model simulation). For unequal choices, the simulated RT showed similar patterns to the

575 observed data, in which choosing between medium and low certainty cues led to the longest RT.

576 For forced choices, similar to the observed data, higher reward certainty and preferred cues were

577 associated with faster RT in simulation. However, simulated RT in forced choices was longer

578 than the experimental data, suggesting that simple reactions to a single cue may engage distinct

579 cognitive processes beyond the current model.

581 EEG results: event-related potentials

582 We focused our EEG analysis on equal choices (with additional control analysis on EEG data

583 from forced choices), because both reward certainty and preference bias played major roles in

584 shaping the behavioral performance of that condition. Trial-average ERPs were formed for each 
585 participant, with epochs time-locked to reward cue onset in equal and forced trials. Different

586 reward certainty levels produced similar grand-average ERP waveforms during equal (Fig. 5A)

587 and forced (Fig. 5B) choices, with a negative peak in the $100-150 \mathrm{~ms}$ time window (the N100

588 component) and a positive peak in the 300 - $400 \mathrm{~ms}$ time window (the P300 component).

589 When assessing the effect of reward certainty on ERPs, we found no univariate differences

590 survived correction for multiple comparisons in equal ( $p>0.552$, cluster-level permutation test

591 across 32 electrodes and all time points) or forced choices $(p>0.175$, cluster-level permutation

592 test). For equal trials, we found no significant difference in ERPs between preferred and non-

593 preferred choices (Fig. 5C, $p>0.208$, cluster-level permutation test). Therefore, in the current

594 study, univariate ERPs were not sensitive to reward certainty or preferred/non-preferred choices.

EEG results: multivariate patterns in equal choices

597 To decode multivariate information of reward certainty in equal choice trials, we applied the

598 linear SVM on multivariate EEG patterns across all electrodes (see Multivariate pattern

599 analysis). The binary classification between high and medium reward certainty was significantly

600 above chance ( $p<0.003$, cluster permutation correction) from $120 \mathrm{~ms}$ after cue onset (Fig. 6A).

601 Similarly, the information between high and low reward certainty was decodable above chance

602 from $132 \mathrm{~ms}$ after cue onset ( $p<0.042$, cluster permutation correction). Relevance spatial

603 patterns based on SVM's weight vector (Haufe et al., 2014) showed that mid-line central and

604 posterior electrodes contained the most information for significant classification. We found no

605 significant classification accuracy between medium and high reward certainty $(p>0.073$ in all

606 time points, uncorrected). 
607 We applied a similar classification procedure to discriminate the information between equal trials

608 in which the participants chose their preferred or non-preferred choices across reward certainty

609 levels. The information about preferred versus non-preferred choices was decodable from 212

$610 \mathrm{~ms}$ to $424 \mathrm{~ms}$ after cue onset ( $p<0.003$, cluster permutation correction).

\section{EEG-informed cognitive modelling}

613 Previous studies supported that the P300 component is associated with evidence accumulation

614 during decision-making (Johnson, 1993; Kok, 2001; Nieuwenhuis et al., 2005; Twomey et al.,

615 2015). Considering that the latency of early visual processing (e.g., N100 peak latency) is a part

616 of non-decision time external to the evidence accumulation process (Nunez et al., 2018), we

617 further hypothesized that the evidence accumulation process initiated at N100 peak latency. This

618 led to a theoretical prediction that the slope of the rise in EEG activity between N100 and P300

619 peak amplitudes reflected the accumulation rate on a trial-by-trial basis. To validate this

620 prediction, we estimated the N100 and P300 components from single trials of equal choices (Fig.

621 7A), using an SVD-based spatial filter to improve the signal-to-noise ratio of single-trial ERPs

622 (see Estimation of single-trial ERP components). This single-trial EEG estimate was then added

623 as a linear regressor (Equation 1) of the mean accumulation rate to the LBA model variant with

624 the best fit to behavioral data (i.e., model 15 in Fig. 3A).

625 We used the same MCMC procedure to fit the extended LBA model with the EEG-informed

626 regressor to the behavioral data of equal choices. The extended LBA model showed good

627 convergence $(\hat{R} \leq 1.02$ for all parameters) and provided a better fit, with a lower LOOIC score

6282687 than the model without the EEG-informed regressor (LOOIC score 2796), suggesting that 
629 the rising slope of N100-P300 indeed affected the decision process. The posterior estimate of the

630 regression coefficient $\beta$ suggested strong evidence for a positive single-trial effect (Fig. 7B, $P_{p \mid \mathrm{D}}$

$631=0.983)$, indicating that a bigger N100-300 slope is associated with faster accumulation rate.

633 Additional analyses: alternative EEG regressors and representations of choice

634 types

635 Is it possible that a simpler EEG-based regressor based on a single ERP component could

636 provide a better model fit than the N100-P300 slope? To test this possibility, we tested four

637 additional extended LBA models with different single-trial EEG regressors applied to the mean

638 accumulation rate: N100 peak latency, N100 peak amplitude, P300 peak latency and P300 peak

639 latency. All the alternative regression models showed inferior fits (LOOIC scores larger than

640 2700) than the one with the regressor of the N100-P300 slope. Therefore, the effects of single-

641 trial EEG activity on the accumulation rate related to multiple ERP components.

642 We did not observe above-chance classification between equal trials with medium and low

643 reward certainty (Fig. 6A). One may concern whether the lack of significant classification was

644 due to the small number of trials in those conditions. To rule out this possibility, we conducted

645 binary classifications to discriminate equal and forced trials with the same reward certainty. The

646 information about trial types (equal vs. forced) was decodable at every level of reward certainty

647 (Fig. 6C, $p<0.05$, cluster corrected), including the one with the least number of trials (i.e., the

648 low reward certainty). This result was expected, given the large difference in stimulus

649 presentation and behavioral performance between the two types of choices. SVM-based

650 relevance patterns highlighted the middle central and frontal electrodes to contain most of the 
651 information of trial types. These results suggested that the difference in classification accuracies

652 between reward certainty levels could not be readily caused by differences in the number of trials.

\section{Discussion}

655 We provided novel evidence that reward certainty and spontaneous preference shape voluntary

656 decision processes and their electrophysiological signatures. When choosing between options

657 with equal reward certainty, higher reward certainty facilitated RT, resulted in a certainty effect.

658 We further observed a preference effect: biasing towards one of the two equal options with a

659 higher choice probability and faster RT. The information of certainty and preference could be

660 reliably decoded from multivariate ERP patterns early during decisions, but not from univariate

661 EEG activities. Using hierarchal Bayesian implementation of a cognitive model, we showed that

662 reward certainty and preference bias were associated with changes in the accumulation rate, a

663 model-derived parameter to account for the speed of evidence accumulation. The accumulation

664 rate was further affected, on a trial-by-trial basis, by the slope of the rise in ERPs between the

665 N100 and P300 components. Together, the current study provided neurocognitive explanations

666 for the behavioral effects during voluntary decisions.

667 The certainty effect on RT persisted from equal choices to simple reactions to cue locations in

668 forced trials (Fig. 2D). In unequal choices, there was also a negative association between RT and

669 the sum of reward certainty of the two choices (Fig. 2E). Therefore, we observed a general

670 tendency of accelerating ones' responses in the presence of more certain reward, even though the

671 reward was not contingent upon the RT. These results were akin to the influence of reward

672 magnitude in humans, which also demonstrated a facilitating effect on RT (Schurman and 
673 Belcher, 1974; Chen and Kwak, 2017). In non-human primates, the phasic activation of

674 dopamine neurons in the ventral midbrain has similar response profiles to changes in reward

675 certainty and magnitude (Fiorillo et al., 2003), suggesting a common mesolimbic dopaminergic

676 pathway underlying different facets of reward processing that affect decision-making.

677 Bayesian model comparison identified specific effects of reward certainty on accumulation rates,

678 highlighting two possible cognitive origins of the certainty effect. First, higher reward certainty

679 resulted in larger mean accumulation rates in equal choices (Fig. 4), consistent with previous

680 studies on perceptual and value-based decisions (Pirrone et al., 2014; Teodorescu et al., 2016).

681 The accumulation rate has been linked to the allocation of attention on the task (Schmiedek et al.,

682 2007). Because reward plays a key role in setting both voluntary (top-down) and stimulus-driven

683 (bottom-up) attentional priority (Libera and Chelazzi, 2006; Raymond and O’Brien, 2009; Krebs

684 et al., 2010; Yantis et al., 2012, Won and Leber, 2016), high reward certainty may boost the

685 attentional resources allocated to sensory processing for more rapid decisions.

686 Second, reward certainty affected the variability of accumulation rates across trials (Fig. 4).

687 Higher accumulation rate variability has been associated with better-memorized items (Starns

688 and Ratcliff, 2014; Osth et al., 2017; Tillman et al., 2017). It is possible that stimuli with higher

689 reward certainty are memorized more strongly (Miendlarzewska et al., 2016), a hypothesis to be

690 confirmed in future studies.

691 Furthermore, MVPA of stimulus-locked ERPs showed multivariate EEG patterns to distinguish

692 between certainty levels as early as $120 \mathrm{~ms}$ after stimulus onset (Fig 5A, see also Thomas et al.,

693 2013), and model comparisons found no evidence to support the non-decision time to vary

694 between certainty levels (Fig. 3A). Considering the average RT of 600-900 ms in equal choices, 
695 our results ruled out the latency of post-decision motor preparation, which constitutes a part of

696 the non-decision time (Karahan et al., 2019), to be the main source of the certainty effect.

697 Interestingly, we observed a monotonic but nonlinear relationship between reward certainty and

698 RT in equal choices: the difference between certain (100\%) and uncertain (80\% and 20\%)

699 reward was greater than that between the two uncertain conditions. This pointed to a special

700 status of the $100 \%$ reward certainty distinct from lower certainty levels, as the latter always

701 carried a non-zero risk of no reward. The salient representation of the $100 \%$ reward certainty was

702 further highlighted by the lack of significant EEG pattern classification between the two lower

703 certainty levels (i.e., $80 \%$ vs. 20\%, Fig. 6A). Here, the certainty effect in rapid voluntary

704 decisions resembles risk-averse behavior in economic decisions (Kahneman and Tversky, 1979),

705 which overweighs outcomes with high reward certainty relative to less probable ones.

706 When choosing between equally valued options, classical evidence accumulation theories

707 predicts a deadlock scenario with a prolonged decision process (Bogacz et al., 2006). This was

708 not supported by recent experimental findings in value-based decisions (Pais et al., 2013; Pirrone

709 et al., 2014), including the current study, in which equal choices took no longer than unequal

710 ones. Our behavioral, modelling and EEG analyses indicated a preference bias, which effectively

711 served as a cognitive mechanism to break the decision deadlock. Compared with non-preferred

712 options, preferred decisions facilitated RTs, associated with larger accumulation rates and

713 evoked distinct EEG multivariate patterns. The preference effect on RT was independent of

714 reward certainty (Fig. 2A) and maintained further in forced trials (Fig. 2D), suggesting its

715 systematical presence as a decision strategy. Because the cue-reward mapping was initially

716 randomized and later changed within each session, this preference bias was not due to stimulus

717 salience but established spontaneously (Voigt et al., 2019). Multiple factors may contribute to the 
718 establishment of preferred options, including simple heuristics or outcomes of early choices that

719 unbalance subjective evaluations of equal cues (Izuma and Murayama, 2013; Bakkour et al.,

720 2018). Future studies could validate these hypotheses by employing more frequent cue-reward

721 re-mapping throughout experiments.

722 Our study highlighted the advantages of EEG-informed cognitive modelling to inform behavioral

723 data. Hierarchical Bayesian parameter estimation of the LBA model provided a robust fit to an

724 individual's behavioral performance with less experimental data needed than other model-fitting

725 methods (Vandekerckhove et al., 2011; Wiecki et al., 2013; Zhang et al., 2016). By integrating

726 single-trial EEG regressors with the cognitive model, we identified the accumulation rate to be

727 affected by the rate of EEG activity changes between visual N100 and P300 components. This

728 results contributed to a growing literature of EEG markers of evidence accumulation processes,

729 including ERP components (Twomey et al., 2015; Loughnane et al., 2016; Nunez et al., 2017),

730 readiness potential (Lui et al., 2018) and oscillatory power (van Vugt et al., 2012). It further

731 consolidated the validity of evidence accumulation as a common computational mechanism

732 leading to voluntary choices of rewarding stimuli (Summerfield and Tsetsos, 2012; Maoz et al.,

733 2019), beyond its common applications to perceptually difficult and temporally extended

734 paradigms.

735 The EEG-informed modelling built upon the known functional link between the P300 component

736 and evidence accumulation for decisions (Polich et al., 1996; Nieuwenhuis et al., 2005; Verleger

737 et al., 2005). A new extension in the current study was to consider the accumulation process

738 begins at the peak latency of the visual N100 component. Theoretically, the delayed initiation of

739 the decision process accounted for information transmission time of $60 \sim 80 \mathrm{~ms}$ from the retina

740 (Schmolesky et al., 1998). Single-unit recording concurred this pre-decision delay, as neurons in 
741 putative evidence accumulation regions exhibited a transient dip and recovery activity

742 independent of decisions, approximately $90 \mathrm{~ms}$ after stimulus onset (Roitman and Shadlen, 2002).

743 Practically, our EEG data had a clear N100 component, and time-resolved MVPA identified

744 significant pattern differences between task conditions at a similar latency. Nevertheless, we

745 used simple stimuli with no perceptual noise. The accumulation process may start later in a trial

746 in experiments involving more complex processing of visual information (Nunez et al., 2018).

747 Further research could dissect the non-decision time (White et al., 2014; Tomassini et al., 2019)

748 and compare latencies of visual encoding across decision tasks and stimuli at different levels of

749 complexity.

750 Two issues require further consideration. First, our cognitive modeling was not meant to

751 reproduce all the rich behavioral features in the data. To obtain sufficient observations for

752 model-fitting, we combined the data before and after cue-reward re-mapping. As a result, our

753 model did not account for behavioural changes related to cue re-mapping. Further study could

754 employ a multi-session design to investigate how learning new cue-reward mappings influence

755 model parameters (Zhang and Rowe, 2014). Second, we focused on the certainty and preference

756 effects by fitting the LBA model only to the data from equal choices. Although simulations

757 indicated that the fitted model provided similar behavioral patterns as in the empirical data in

758 unequal and forced choices, it was not fitted directly to the experiment data in those two choice

759 conditions. A more parsimonious model for all three types of choices would require additional

760 assumptions, which is beyond the scope of the current study. For example, to incorporate the

761 large RT discrepancy between equal and forced choices, one could assume that the urgency

762 signal (Boehm et al., 2016; Thura and Cisek, 2017) plays a more dominant role in accelerating

763 RT when no apparent comparisons are needed in forced choices. 
764 In conclusion, when choosing between equal valued options, reward certainty and preference

765 bias selectively modulated decision processes and their electrophysiological signatures. These

766 findings extended and substantiated the computational framework of evidence accumulation for

767 voluntary decisions. Our results further highlighted the intricate nature of human behavior

768 susceptible to external factors as well as endogenous heuristics.

770 References

771 Abler B, Herrnberger B, Grön G, Spitzer M (2009) From uncertainty to reward: BOLD 772 characteristics differentiate signaling pathways. BMC Neurosci 10:154.

773 Ahn W-Y, Haines N, Zhang L (2017) Revealing Neurocomputational Mechanisms of 774 Reinforcement Learning and Decision-Making With the hBayesDM Package. Comput $775 \quad$ psychiatry (Cambridge, Mass) 1:24-57.

776 Annis J, Miller BJ, Palmeri TJ (2017) Bayesian inference with Stan: A tutorial on adding custom 777 distributions. Behav Res Methods 49:863-886.

778 Baayen RH, Davidson DJ, Bates DM (2008) Mixed-effects modeling with crossed random 779 effects for subjects and items. J Mem Lang 59:390-412.

780 Bagiella E, Sloan RP, Heitjan DF (2000) Mixed-effects models in psychophysiology. $781 \quad$ Psychophysiology 37:13-20.

782 Bakkour A, Zylberberg A, Shadlen MN, Shohamy D (2018) Value-based decisions involve 783 sequential sampling from memory. bioRxiv 269290.

784 Ballard IC, Kim B, Liatsis A, Aydogan G, Cohen JD, McClure SM (2017) More Is Meaningful: 

28:1443-1454.

Barr DJ, Levy R, Scheepers C, Tily HJ (2013) Random effects structure for confirmatory hypothesis testing: Keep it maximal. J Mem Lang 68:255-278.

Bates D, Kliegl R, Vasishth S, Baayen H (2015a) Parsimonious Mixed Models. ArXiv 1506.04967.

Bates D, Mächler M, Bolker BM, Walker SC (2015b) Fitting linear mixed-effects models using lme4. J Stat Softw 67.

Bem DJ (1967) Self-perception: An alternative interpretation of cognitive dissonance phenomena. Psychol Rev 74:183-200. 19:58-80. Impatience in perceptual decision-making. Psychon Bull Rev 23:738-749. making: a formal analysis of models of performance in two-alternative forced-choice tasks.

803 Brown SD, Heathcote A (2008) The simplest complete model of choice response time: linear ballistic accumulation. Cogn Psychol 57:153-178.

805 Brunton BW, Botvinick MM, Brody CD (2013) Rats and Humans Can Optimally Accumulate 
Evidence for Decision-Making. Science (80- ) 340:95-98.

807 Carpenter B, Gelman A, Hoffman MD, Lee D, Goodrich B, Betancourt M, Brubaker M, Guo J, 808 Li P, Riddell A (2017) Stan $\square$ : A Probabilistic Programming Language. J Stat Softw 76.

809 Cavanagh JF, Wiecki T V, Cohen MX, Figueroa CM, Samanta J, Sherman SJ, Frank MJ (2011)

810 Subthalamic nucleus stimulation reverses mediofrontal influence over decision threshold.

$811 \quad$ Nat Neurosci 14:1462-1467.

812 Chen MK, Risen JL (2010) How Choice Affects and Reflects Preferences: Revisiting the Free-

813 Choice Paradigm. J Pers Soc Psychol 99:573-594.

814 Chen X-J, Kwak Y (2017) What Makes You Go Faster?: The Effect of Reward on Speeded $815 \quad$ Action under Risk. Front Psychol 8:1057.

816 Cichy RM, Pantazis D, Oliva A (2014) Resolving human object recognition in space and time. $817 \quad$ Nat Neurosci 17:455-462.

818 Delorme A, Makeig S (2004) EEGLAB $\square$ : an open source toolbox for analysis of single-trial 819 EEG dynamics including independent component analysis. 134:9-21.

820 Dima DC, Perry G, Messaritaki E, Zhang J, Singh KD (2018) Spatiotemporal dynamics in 821 human visual cortex rapidly encode the emotional content of faces. Hum Brain Mapp 822 39:3993-4006.

823 Ditterich J, Mazurek ME, Shadlen MN (2003) Microstimulation of visual cortex affects the 824 speed of perceptual decisions. Nat Neurosci 6:891-898.

825 Festinger L (1957) A theory of cognitive dissonance. A theory Cogn dissonance:xi, 291-xi, 291.

826 Fiorillo CD, Tobler PN, Schultz W (2003) Discrete Coding of Reward Probability and 
Uncertainty by Dopamine Neurons. Science (80- ) 299:1898 LP - 1902.

828 Forstmann BU, Anwander A, Schäfer A, Neumann J, Brown S, Wagenmakers E-J, Bogacz R,

829 Turner R (2010a) Cortico-striatal connections predict control over speed and accuracy in perceptual decision making. Proc Natl Acad Sci U S A 107:15916-15920.

831 Forstmann BU, Brown S, Dutilh G, Neumann J, Wagenmakers E-J (2010b) The neural substrate of prior information in perceptual decision making: a model-based analysis. Front Hum

Garrett D, Peterson DA, Anderson CW, Thaut MH (2003) Comparison of linear, nonlinear, and Neurosci 4:40.

Gelman A, Rubin DB (1992) Inference from Iterative Simulation Using Multiple Sequences. Stat Sci 7:457-472.

Gold JI, Shadlen MN (2001) Neural computations that underlie decisions about sensory stimuli. Trends Cogn Sci 5:10-16. 574.

843 Gueorguieva R, Krystal JH (2004) Move over ANOVA: progress in analyzing repeated844 measures data and its reflection in papers published in the Archives of General Psychiatry. $845 \quad$ Arch Gen Psychiatry 61:310-317.

846 Haufe S, Meinecke F, Görgen K, Dähne S, Haynes JD, Blankertz B, Bießmann F (2014) On the interpretation of weight vectors of linear models in multivariate neuroimaging. Neuroimage 
$87: 96-110$.

849 Hawkins GE, Wagenmakers E-J, Ratcliff R, Brown SD (2015) Discriminating evidence 850 accumulation from urgency signals in speeded decision making. J Neurophysiol 114:40-47.

851 Heekeren HR, Marrett S, Ungerleider LG (2008) The neural systems that mediate human 852 perceptual decision making. Nat Rev Neurosci 9:467-479.

853 Ho TC, Brown S, Serences JT (2009) Domain general mechanisms of perceptual decision 854 making in human cortex. J Neurosci 29:8675-8687.

855 Izuma K, Matsumoto M, Murayama K, Samejima K, Sadato N, Matsumoto K (2010) Neural 856 correlates of cognitive dissonance and choice-induced preference change. Proc Natl Acad 857 Sci U S A 107:22014-22019.

858 Izuma K, Murayama K (2013) Choice-Induced Preference Change in the Free-Choice Paradigm: 859 A Critical Methodological Review. Front Psychol 4:1-12.

860 Jahfari S, Ridderinkhof KR, Scholte HS (2013) Spatial Frequency Information Modulates 861 Response Inhibition and Decision-Making Processes Chacron MJ, ed. PLoS One 8:e76467.

862 Johnson R (1993) On the neural generators of the P300 component of the event-related potential.

$863 \quad$ Psychophysiology 30:90-97.

864 Kahneman D, Tversky A (1979) Prospect Theory: An Analysis of Decision under Risk. $865 \quad$ Econometrica 47:263-291.

866 Karahan E, Costigan AG, Graham KS, Lawrence AD, Zhang J (2019) Cognitive and White867 Matter Compartment Models Reveal Selective Relations between Corticospinal Tract 868 Microstructure and Simple Reaction Time. J Neurosci 39:5910-5921. 
Kayser J, Tenke CE (2003) Optimizing PCA methodology for ERP component identification and measurement: Theoretical rationale and empirical evaluation. Clin Neurophysiol 114:2307-

Kelly SP, O'Connell RG (2013) Internal and External Influences on the Rate of Sensory 2325.

Kok A (2001) On the utility of P3 amplitude as a measure of processing capacity. Evidence Accumulation in the Human Brain. J Neurosci 33:19434-19441.

Krebs RM, Boehler CN, Woldorff MG (2010) The influence of reward associations on conflict processing in the Stroop task. Cognition 117:341-347.

Libera C Della, Chelazzi L (2006) Visual Selective Attention and the Effects of Monetary Rewards. Psychol Sci 17:222-227. selection signals influence perceptual decisions by modulating the onset and rate of readiness potentials reflect a decision-making process in the human brain. bioRxiv Prepr evidence accumulation. Curr Biol 26:496-502.

Mangun GR, Hillyard SA (1991) Modulations of sensory-evoked brain potentials indicate 
Perform 17:1057-1074.

891 Maoz U, Yaffe G, Koch C, Mudrik L (2019) Neural precursors of deliberate and arbitrary 892 decisions in the study of voluntary action. bioRxiv:97626.

893 Maris E, Oostenveld R (2007) Nonparametric statistical testing of EEG- and MEG-data. J $894 \quad$ Neurosci Methods 164:177-190.

895 Matuschek H, Kliegl R, Vasishth S, Baayen H, Bates D (2017) Balancing Type I error and power 896 in linear mixed models. J Mem Lang 94:305-315.

897 Miendlarzewska EA, Bavelier D, Schwartz S (2016) Neuroscience and Biobehavioral Reviews

898 Influence of reward motivation on human declarative memory. Neurosci Biobehav Rev $899 \quad 61: 156-176$.

900 Mulder MJ, Bos D, Weusten JMH, van Belle J, van Dijk SC, Simen P, van Engeland H, Durston

901 S (2010) Basic impairments in regulating the speed-accuracy tradeoff predict symptoms of 902 attention-deficit/hyperactivity disorder. Biol Psychiatry 68:1114-1119.

903 Mulder MJ, van Maanen L, Forstmann BU (2014) Perceptual decision neurosciences - a model$904 \quad$ based review. Neuroscience 277:872-884.

905 Nieuwenhuis S, Aston-Jones G, Cohen JD (2005) Decision making, the P3, and the locus 906 coeruleus-norepinephrine system. Psychol Bull 131:510-532.

907 Nunez MD, Gosai A, Vandekerckhove J, Srinivasan R (2018) The latency of a visual evoked 908 potential tracks the onset of decision making. bioRxiv:275727.

909 Nunez MD, Vandekerckhove J, Srinivasan R (2017) How attention influences perceptual 910 decision making: Single-trial EEG correlates of drift-diffusion model parameters. J Math 
Psychol 76:117-130.

912 Osth AF, Dennis S, Heathcote A (2017) Likelihood ratio sequential sampling models of 913 recognition memory. Cogn Psychol 92:101-126.

914 Oud B, Krajbich I, Miller K, Cheong JH, Botvinick M, Fehr E (2016) Irrational time allocation

915 in decision-making. Proc R Soc B Biol Sci 283:20151439.

916 Pais D, Hogan PM, Schlegel T, Franks NR, Leonard NE, Marshall J a R (2013) A mechanism for $917 \quad$ value-sensitive decision-making. PLoS One 8:e73216.

918 Parra LC, Spence CD, Gerson AD, Sajda P (2005) Recipes for the linear analysis of EEG.

$919 \quad$ Neuroimage 28:326-341.

920 Pirrone A, Azab H, Hayden BY, Stafford T, Marshall JAR (2018) Evidence for the speed-value

921 trade-off: Human and monkey decision making is magnitude sensitive. Decision 5:129-142.

922 Pirrone A, Stafford T, Marshall JAR (2014) When natural selection should optimize speed923 accuracy trade-offs. Front Neurosci 8:1-5.

924 Polich J, Ellerson PC, Cohen J (1996) P300, stimulus intensity, modality, and probability. Int J $925 \quad$ Psychophysiol 23:55-62.

926 Ratcliff R, Smith PL (2004) A comparison of sequential sampling models for two-choice 927 reaction time. Psychol Rev 111:333-367.

928 Raymond JE, O’Brien JL (2009) Selective Visual Attention and Motivation: The Consequences 929 of Value Learning in an Attentional Blink Task. Psychol Sci 20:981-988.

930 Roitman JD, Shadlen MN (2002) Response of neurons in the lateral intraparietal area during a 931 combined visual discrimination reaction time task. J Neurosci 22:9475-9489. 
932 Rustichini A (2009) Neuroeconomics: Formal models of decision making and cognitive

933 neuroscience. In: Neuroeconomics, pp 33-46.

934 Schiebener J, Brand M (2015) Decision Making Under Objective Risk Conditions-a Review of

935 Cognitive and Emotional Correlates, Strategies, Feedback Processing, and External

936 Influences. Neuropsychol Rev 25:171-198.

937 Schmiedek F, Oberauer K, Wilhelm O, Süss H-M, Wittmann WW (2007) Individual differences

938 in components of reaction time distributions and their relations to working memory and

939 intelligence. J Exp Psychol Gen 136:414-429.

940 Schmolesky MT, Wang Y, Hanes DP, Thompson KG, Leutgeb S, Schall JD, Leventhal AG

941 (1998) Signal Timing Across the Macaque Visual System. J Neurophysiol 79:3272-3278.

942 Schmolesky MT, Wang Y, Hanes DP, Thompson KG, Leutgeb S, Schall JD, Leventhal AG

943 (2017) Signal Timing Across the Macaque Visual System. J Neurophysiol 79:3272-3278.

944 Schurman DL, Belcher JP (1974) Noncontingent reward magnitude effects on reaction time: A

945 replication and extension. Bull Psychon Soc 3:104-106.

946 Smith PL, Ratcliff R (2004) Psychology and neurobiology of simple decisions. Trends Neurosci

$947 \quad 27: 161-168$.

948 Starns JJ, Ratcliff R (2014) Validating the unequal-variance assumption in recognition memory

949 using response time distributions instead of ROC functions: A diffusion model analysis. J

$950 \quad$ Mem Lang 70:36-52.

951 Summerfield C, Tsetsos K (2012) Building Bridges between Perceptual and Economic Decision-

952 Making: Neural and Computational Mechanisms. Front Neurosci 6:70. 
953 Teodorescu AR, Moran R, Usher M (2016) Absolutely relative or relatively absolute: violations

954 of value invariance in human decision making. Psychon Bull Rev 23:22-38.

955 Thaler R (1981) Some empirical evidence on dynamic inconsistency. Econ Lett 8:201-207.

956 Thomas J, Vanni-Mercier G, Dreher J-C (2013) Neural dynamics of reward probability coding: a

957 Magnetoencephalographic study in humans. Front Neurosci 7:214.

958 Thura D, Cisek P (2017) The Basal Ganglia Do Not Select Reach Targets but Control the

$959 \quad$ Urgency of Commitment. Neuron 95:1160-1170.e5.

960 Tillman G, Osth AF, van Ravenzwaaij D, Heathcote A (2017) A diffusion decision model 961 analysis of evidence variability in the lexical decision task. Psychon Bull Rev 24:19499621956.

963 Tobler PN, O’Doherty JP, Dolan RJ, Schultz W (2007) Reward value coding distinct from risk 964 attitude-related uncertainty coding in human reward systems. J Neurophysiol 97:1621-1632.

965 Tomassini A, Price D, Zhang J, Rowe JB (2019) On the evolution of neural decisions from 966 uncertain visual input to uncertain actions. bioRxiv:803049.

967 Twomey DM, Murphy PR, Kelly SP, O’Connell RG (2015) The classic P300 encodes a build-to968 threshold decision variable. Eur J Neurosci 42:1636-1643.

969 van Vugt MK, Simen P, Nystrom LE, Holmes P, Cohen JD (2012) EEG oscillations reveal 970 neural correlates of evidence accumulation. Front Neurosci 6:106.

971 Vandekerckhove J, Tuerlinckx F, Lee MD (2011) Hierarchical diffusion models for two-choice 972 response times. Psychol Methods 16:44-62.

973 Vehtari A, Gelman A, Gabry J (2017) Practical Bayesian model evaluation using leave-one-out 
cross-validation and WAIC. Stat Comput 27:1413-1432.

975 Verleger R, Jaśkowski P, Wascher E (2005) Evidence for an integrative role of P3b in linking 976 reaction to perception. J Psychophysiol 19:165-181.

977 Voigt K, Murawski C, Speer S, Bode S (2019) Hard Decisions Shape the Neural Coding of $978 \quad$ Preferences. J Neurosci 39:718-726.

979 Wheeler DR (1974) Brand loyalties: qualitative, quantitative, or both? J Acad Mark Sci 2:651$980 \quad 658$.

981 White CN, Congdon E, Mumford JA, Karlsgodt KH, Sabb FW, Freimer NB, London ED, 982 Cannon TD, Bilder RM, Poldrack RA (2014) Decomposing decision components in the 983 stop-signal task: a model-based approach to individual differences in inhibitory control. J $984 \quad$ Cogn Neurosci 26:1601-1614.

985 Wiecki T V, Sofer I, Frank MJ (2013) HDDM: Hierarchical Bayesian estimation of the Drift986 Diffusion Model in Python. Front Neuroinform 7:14.

987 Won BY, Leber A (2016) How do magnitude and frequency of monetary reward guide visual 988 search? Attention, Perception, Psychophys 78.

989 Zhang H, Wang Z (2011) A Normal Distribution-Based Over-Sampling Approach to Imbalanced

990 Data Classification. In: Advanced Data Mining and Applications. ADMA 2011. Lecture $991 \quad$ Notes in Computer Science, vol 7120 (Tang J, King I, Chen L, Wang J, eds), pp 83-96. 992 Springer Berlin / Heidelberg.

993 Zhang J (2012) The Effects of Evidence Bounds on Decision-Making: Theoretical and Empirical 994 Developments. Front Psychol 3:263. 
995 Zhang J, Hughes LE, Rowe JB (2012) Selection and inhibition mechanisms for human voluntary

996 action decisions. Neuroimage 63:392-402.

997 Zhang J, Rittman T, Nombela C, Fois A, Coyle-Gilchrist I, Barker RA, Hughes LE, Rowe JB

998 (2016) Different decision deficits impair response inhibition in progressive supranuclear

$999 \quad$ palsy and Parkinson's disease. Brain 139:161-173.

1000 Zhang J, Rowe JB (2014) Dissociable mechanisms of speed-accuracy tradeoff during visual

1001 perceptual learning are revealed by a hierarchical drift-diffusion model. Front Neurosci 8:69.

1002 Zhang J, Rowe JB (2015) The neural signature of information regularity in temporally extended

1003 event sequences. Neuroimage 107:266-276.

1004 


\section{Tables}

1006 Table 1. The linear mixed-effects models of RT. Model 1 analyzed single-trial RT in equal and

1007 forced trials. Model 2 analyzed single-trial RT in unequal trials. In both models, preference was

1008 a predictor indicating whether the preferred cue was selected in each trial. Cue-remapping was a

1009 predictor indicating whether each trial was before or after cure-reward re-mapping in the second

1010 half of each session. Right-bias indicated whether the cue on the right size of the screen was

1011 chosen in each trial, modeling a possible response bias.

\begin{tabular}{|c|c|c|}
\hline & Model 1 (equal and forced trials) & Model 2 (unequal trials) \\
\hline $\begin{array}{r}\text { Dependent } \\
\text { variables }\end{array}$ & RT & RT \\
\hline Main effects & $\begin{array}{l}\text { reward certainty } \\
\text { preference } \\
\text { cue-remapping } \\
\text { choice (equal or forced) } \\
\text { right-bias }\end{array}$ & $\begin{array}{l}\text { sum of reward certainty } \\
\text { difference of reward certainty } \\
\text { preference } \\
\text { cue-remapping } \\
\text { right-bias }\end{array}$ \\
\hline Interaction terms & $\begin{array}{l}\text { certainty } * \text { choice } \\
\text { certainty } * \text { preference } \\
\text { certainty } * \text { cue-remapping } \\
\text { choice } * \text { preference } \\
\text { choice } * \text { cue-remapping } \\
\text { preference * cue-remapping } \\
\text { certainty } * \text { choice } * \text { preference } \\
\text { certainty } * \text { choice } * \text { cue-remapping } \\
\text { certainty } * \text { cue-remapping } * \text { preference }\end{array}$ & $\begin{array}{l}\text { sum of reward certainty } * \text { preference } \\
\text { difference of reward certainty } * \text { preference } \\
\text { sum of reward certainty } * \text { cue-remapping } \\
\text { difference of reward certainty } * \text { cue-remapping } \\
\text { preference } * \text { cue-remapping }\end{array}$ \\
\hline $\begin{array}{r}\text { Random effects } \\
\text { (correlated slopes } \\
\text { and intercepts) }\end{array}$ & $\begin{array}{l}\text { reward certainty } \\
\text { preference } \\
\text { cue-remapping } \\
\text { choice } \\
\text { right-bias }\end{array}$ & $\begin{array}{l}\text { sum of reward certainty } \\
\text { difference of reward certainty } \\
\text { preference } \\
\text { cue-remapping } \\
\text { right-bias }\end{array}$ \\
\hline
\end{tabular}




\section{Figure 1}

bioRxiv preprint doi: https://doi.org/10.1101/832311; this version posted November 7, 2019. The copyright holder for this preprint (which was not certified by peer review) is the author/funder, who has granted bioRxiv a license to display the preprint in perpetuity. It is made available under aCC-BY-NC 4.0 International license.

A

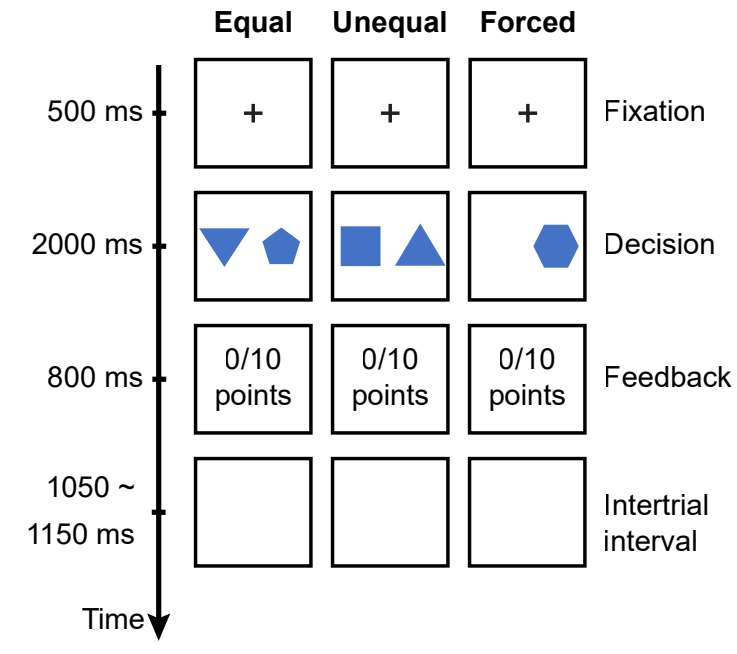

B

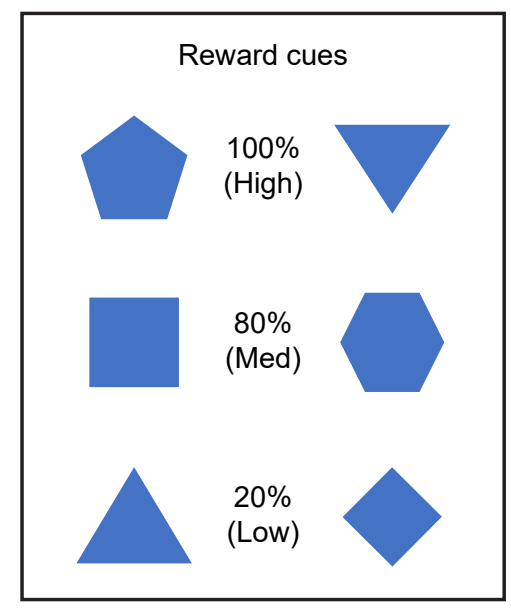

C

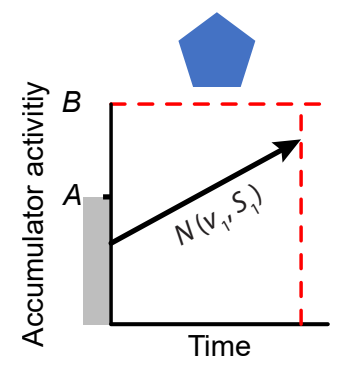

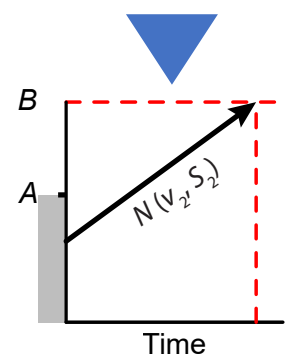

Figure 1. (A) Experimental paradigm of the probabilistic reward task. Participants were instructed to decide between two reward cues (equal and unequal trials) or respond to a single cue (forced trials). (B) A total of six reward cues were randomly assigned to three levels of reward certainty $(100 \%, 80 \%$ or $20 \%)$. (C) Exemplar time course of the linear ballistic accumulator (LBA) model for equal choices. On each trial, the LBA assumes that evidence for two options are accumulated linearly and independently over time in two accumulators. The accumulation rate is sampled from a normal distribution with mean $v$ and standard deviation $S$. The starting point of the accumulation process is sampled from a uniform distribution between 0 and $A$. The accumulation process terminates once the accumulated evidence first reaches a threshold $B$, and a corresponding decision is made by the winning accumulator. 


\section{Figure 2}

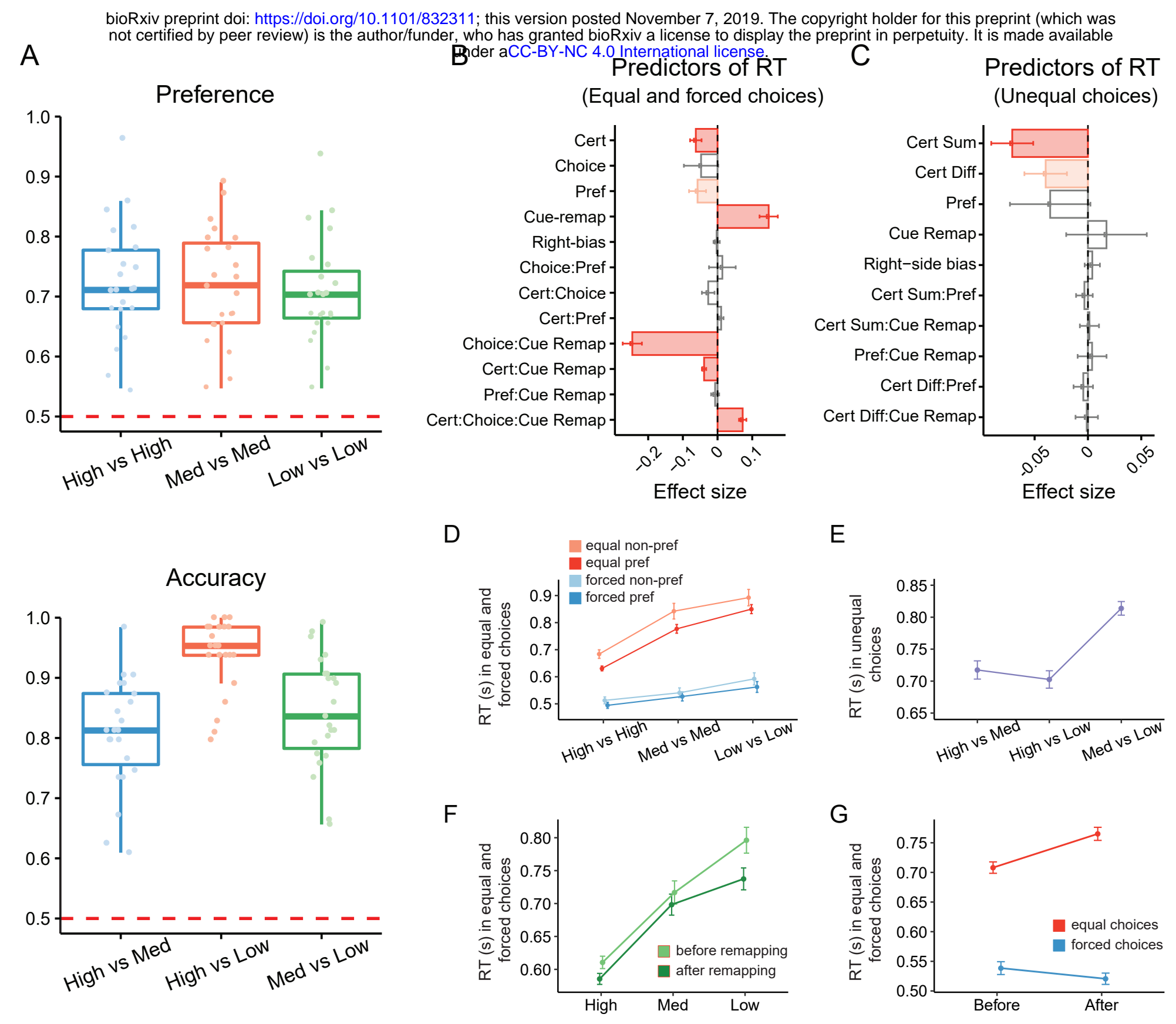

Figure 2. Behavioral results. (A) Preference bias across reward certainty levels in equal trials (top) and decision accuracy across reward certainty levels in unequal trials (bottom). (B) Linear mixed-effects model results for Model 1 in Table 1. Dark red bars represent significant effects with $p<0.001$. Light red bars represent significant effects with $p<0.05$. Grey bars represent nonsignificant factors and interactions. Error bars represent standard errors across participants. (C) Linear mixed-effects model results for Model 2 in Table 1. Significant effects and interactions in RT from Model 1 (Table 1) were presented separately for: reward certainty and preference in equal and forced trials (D), before and after cue-remapping at different reward certainty levels (F), before and after cue-remapping in equal and forced trials $(\mathbf{G})$. Significant main effects in Model 2 were presented in panel E. In panels D-G, Error bars represent standard errors across participants. 


\section{Figure 3}

bioRxiv preprint doi: https://doi.org/10.1101/832311; this version posted November 7, 2019. The copyright holder for this preprint (which was not certified by peer review) is the author/funder, who has granted bioRxiv a license to display the preprint in perpetuity. It is made available under aCC-BY-NC 4.0 International license.

A

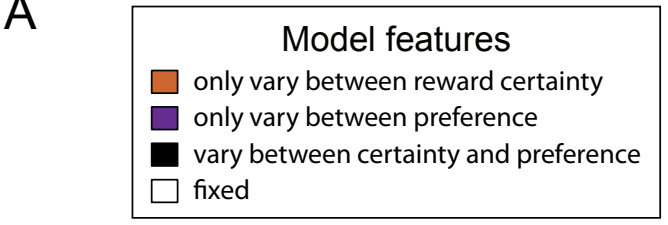

$\checkmark \mathrm{S}$ Ter

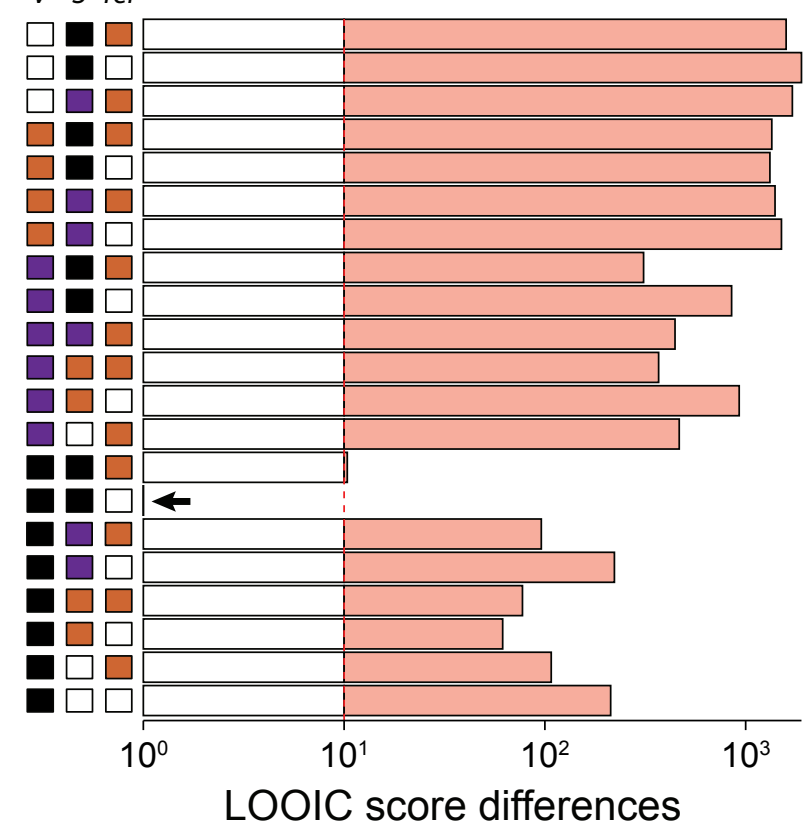

B
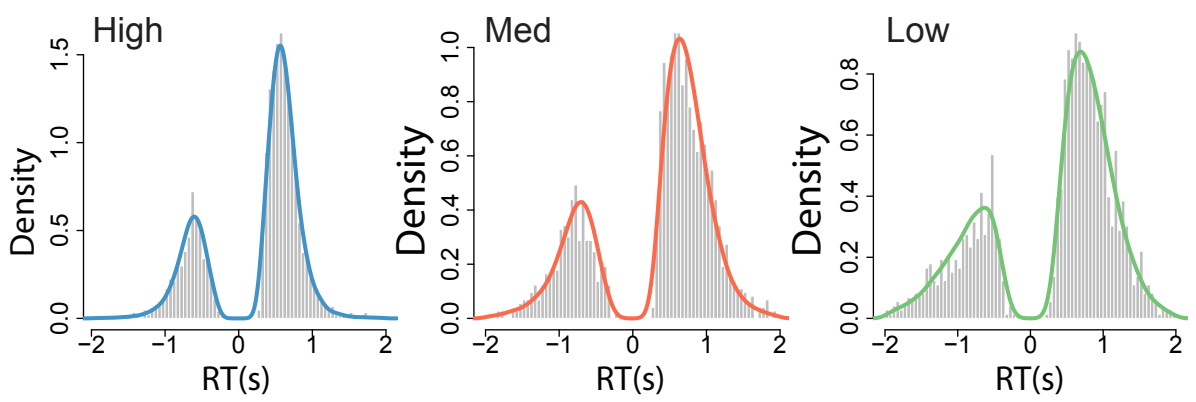

C
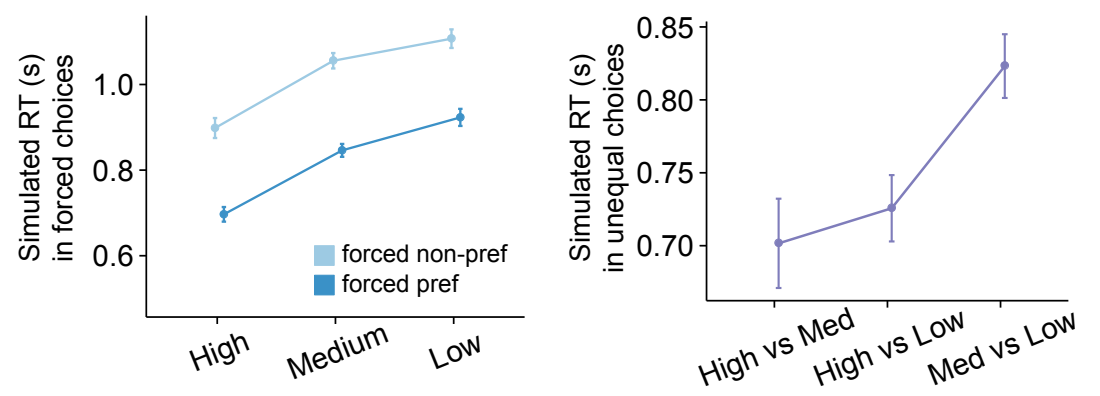

Figure 3. Model comparisons, model fits and model simulations. (A) LOOIC scores of 21 LBA model variants. The LOOIC score differences between all models and the best model are plotted against corresponding model structures, which were illustrated on the left of the panel. The model structure specified how the mean accumulation rate $v$, the standard deviation $S$ of the accumulation rate and the non-decision time $T_{e r}$ could vary between conditions. A black filled square indicated that the corresponding parameter could vary between reward certainty levels and preferred/ non-preferred options. An orange or purple filled square indicated that the corresponding parameter could only vary between reward certainty levels or preferred/non-preferred options, respectively. Unfilled squares indicated that the parameter remained fixed between conditions. The best model was shown with a LOOIC score difference of zero (indicated by the arrow). (B) Simulations of RTs in equal choices, generated from the posterior distribution of the best fitted model for high (left), medium (middle) and low (right) reward certainty levels. Histograms represent experimental data and density distributions represent model simulation from 100 iterations. Negative values represent RTs for non-preferred choices. (C) Simulation of RTs in forced (left) and unequal (right) choices from 100 iterations. Error bars represent standard errors across participants. 


\section{Figure 4}

bioRxiv preprint doi: https://doi.org/10.1101/832311; this version posted November 7, 2019. The copyright holder for this preprint (which was not certified by peer review) is the author/funder, who has granted bioRxiv a license to display the preprint in perpetuity. It is made available under aCC-BY-NC 4.0 International license.

A

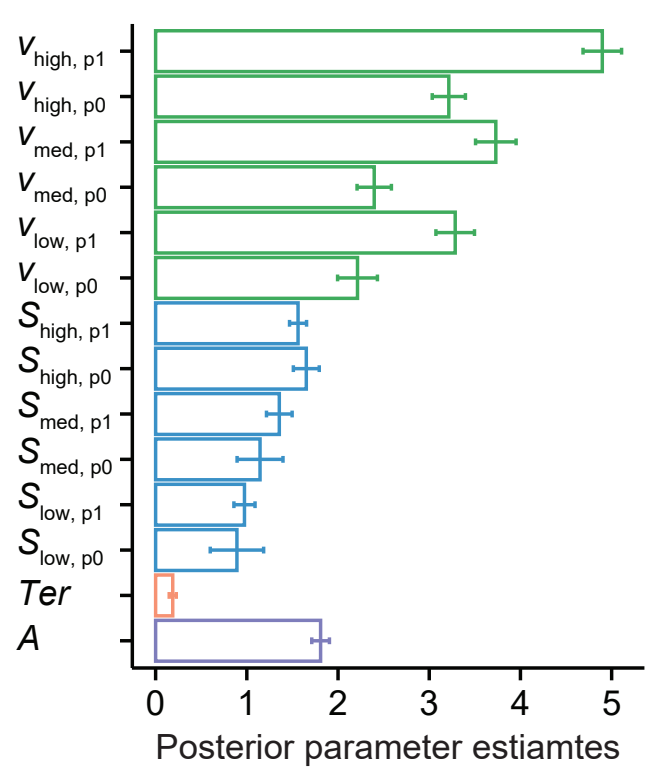

B

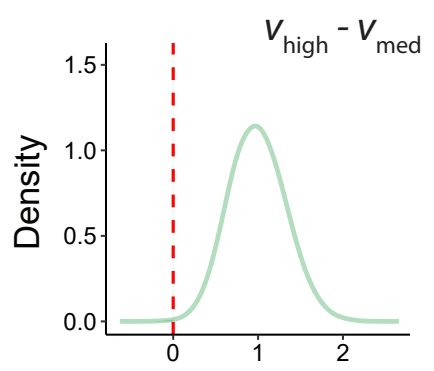

Certainty
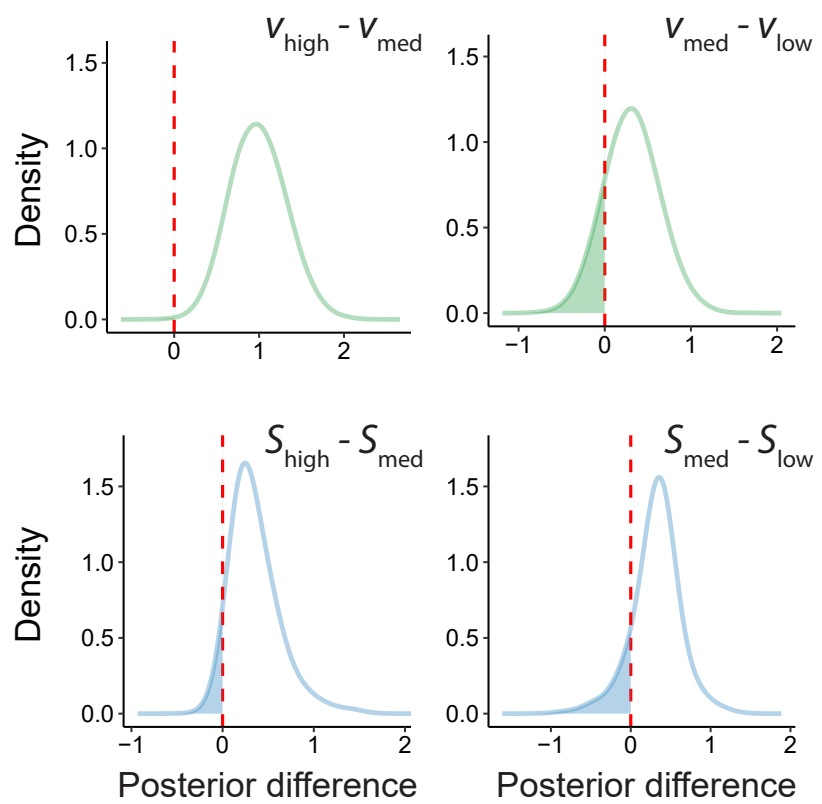

Preference

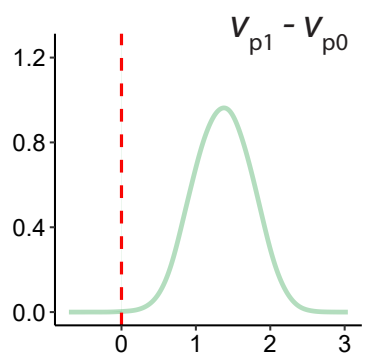

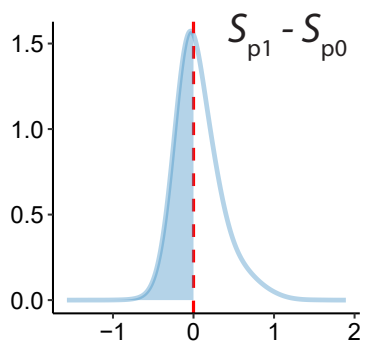

Posterior difference

Figure 4. Posterior model parameters and inferences. (A) Group-level LBA model parameters of the best fitting model: means of accumulation rates ( $v$, green), standard deviations of accumulation rates $\left(S\right.$, blue), non-decision time $\left(T_{e r}\right.$; orange) and starting point $(A$, purple). Error bars represent standard deviations of posterior distributions of parameter values. The means and standard deviations of accumulation rates were shown separately for each reward certainty level (high, medium and low) and accumulator (p1, preferred option; p0, non-preferred option). (B) Differences of posterior parameter estimates across certainty levels (left and middle columns) and preference levels (right column). The proportion of posterior difference distributions above zero suggested higher parameter values for higher certainty level or more preferred options. 


\section{Figure 5}

bioRxiv preprint doi: https://doi.org/10.1101/832311; this version posted November 7, 2019. The copyright holder for this preprint (which was not certified by peer review) is the author/funder, who has granted bioRxiv a license to display the preprint in perpetuity. It is made available under aCC-BY-NC 4.0 International license.
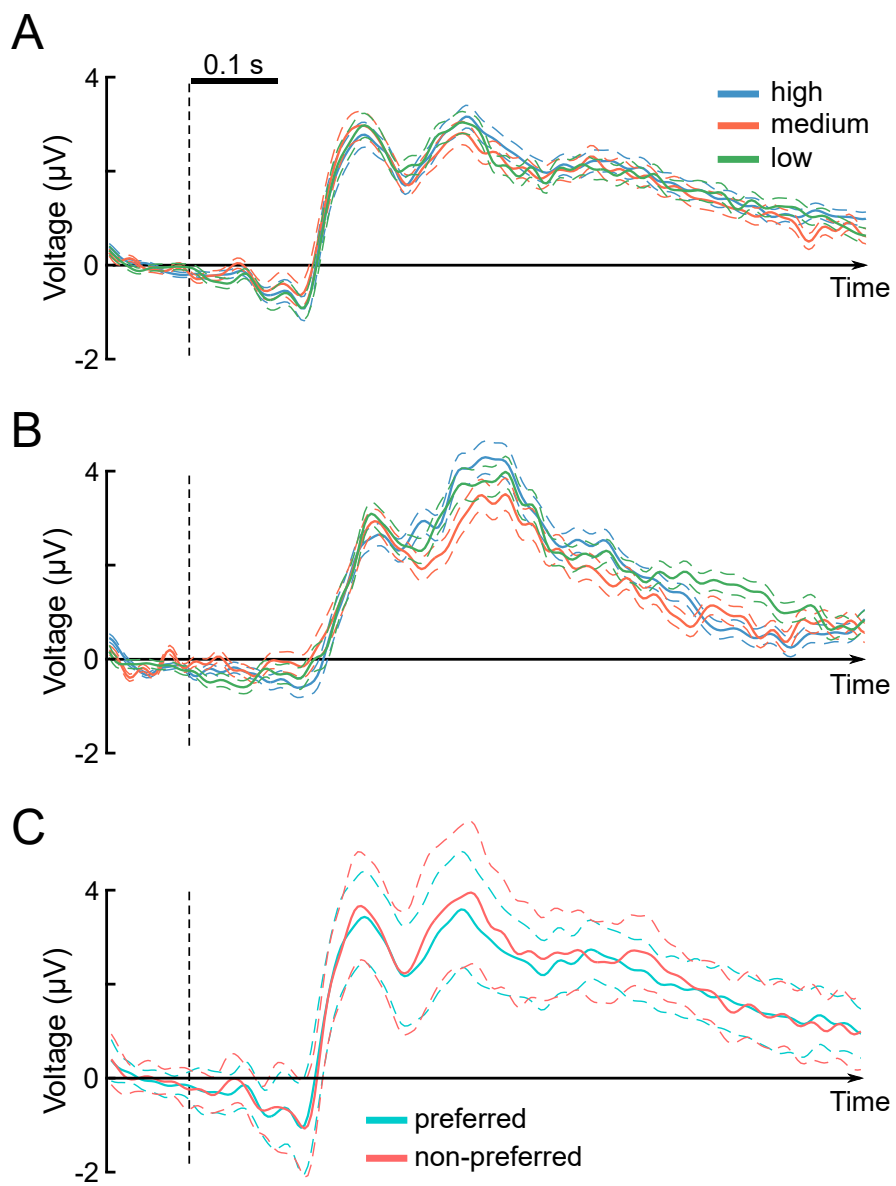

Figure 5. Grand-average stimulus-locked ERPs across all EEG electrodes. (A) ERPs from high (100\%), medium (80\%) and low (20\%) reward certainty in equal trials. (B) ERPs from high (100\%), medium (80\%) and low (20\%) reward certainty in forced trials. (C) ERPs from equal trials in which the preferred or non-preferred cue was chosen. In all panels, the dashed lines represent standard errors across participants. 


\section{Figure 6}

bioRxiv preprint doi: https://doi.org/10.1101/832311; this version posted November 7, 2019. The copyright holder for this preprint (which was not certified by peer review) is the author/funder, who has granted bioRxiv a license to display the preprint in perpetuity. It is made available

A
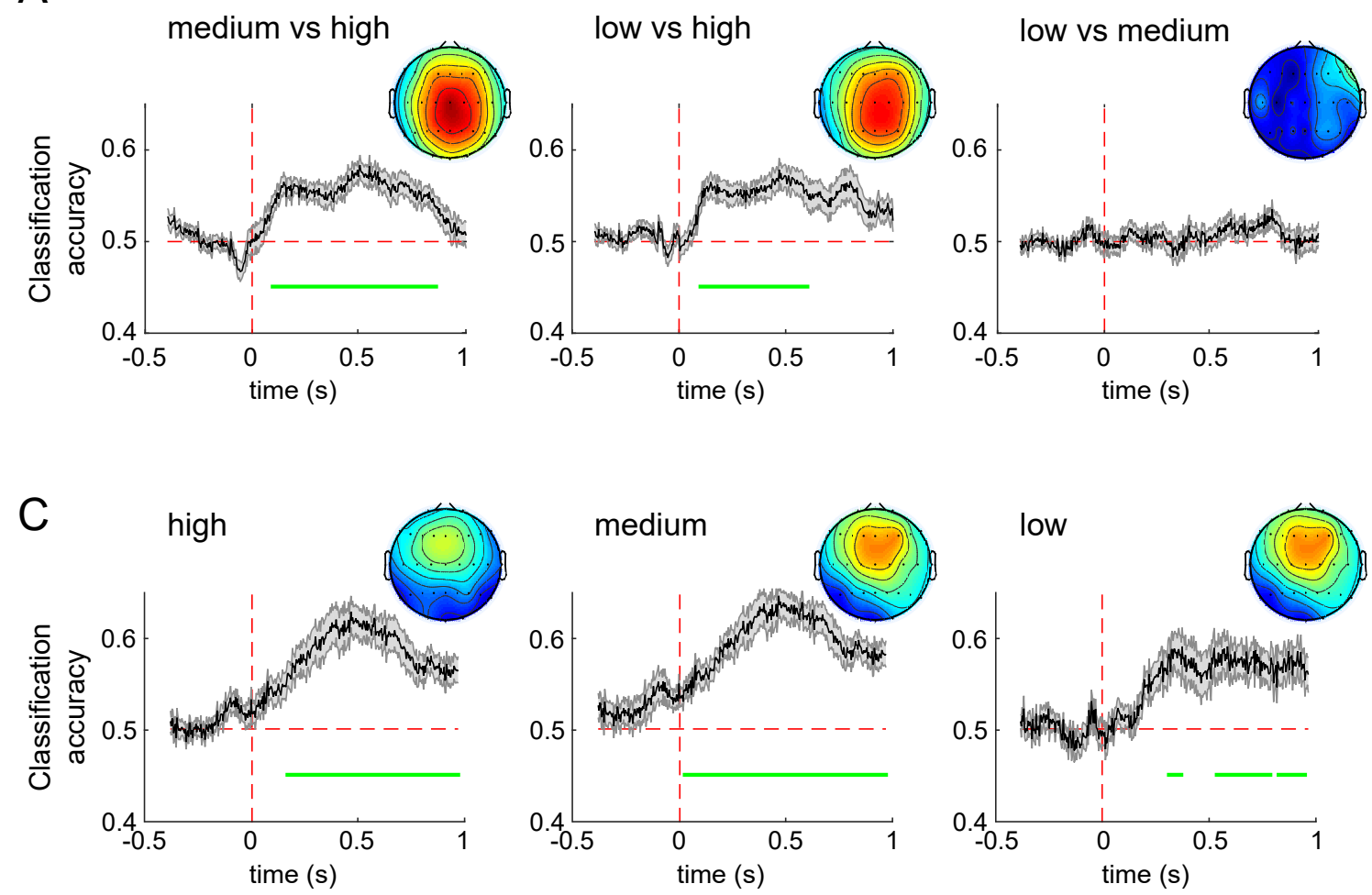

B

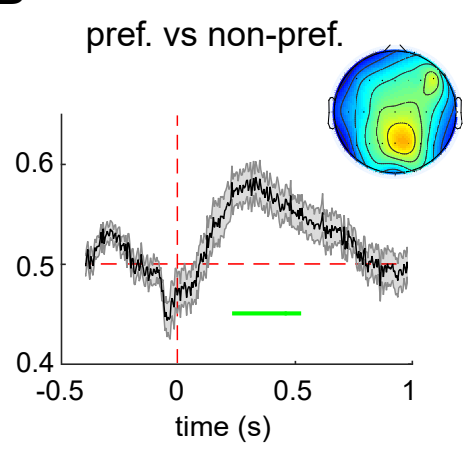

Figure 6. MVPA results. (A) Classification accuracies across time-points between equal choices with different levels of reward certainty. (B) Classification accuracies across time-points between equal trials with preferred and non-preferred choices. (C) Classification accuracies across time-points between equal and forced choices with the same level of reward certainty. In all panels, the black lines denote classification accuracies from a stratified 10-fold cross-validation and the gray areas denote standard errors. Significant decoding time windows (green horizontal bars) were determined from cluster-level permutation tests $(p<0.05$, corrected). Topographic maps represent activation patterns from classification weights, which indicate the contribution of different EEG channels to overall classification accuracies. 


\section{Figure 7}

bioRxiv preprint doi: https://doi.org/10.1101/832311; this version posted November 7, 2019. The copyright holder for this preprint (which was not certified by peer review) is the author/funder, who has granted bioRxiv a license to display the preprint in perpetuity. It is made available under aCC-BY-NC 4.0 International license.

A

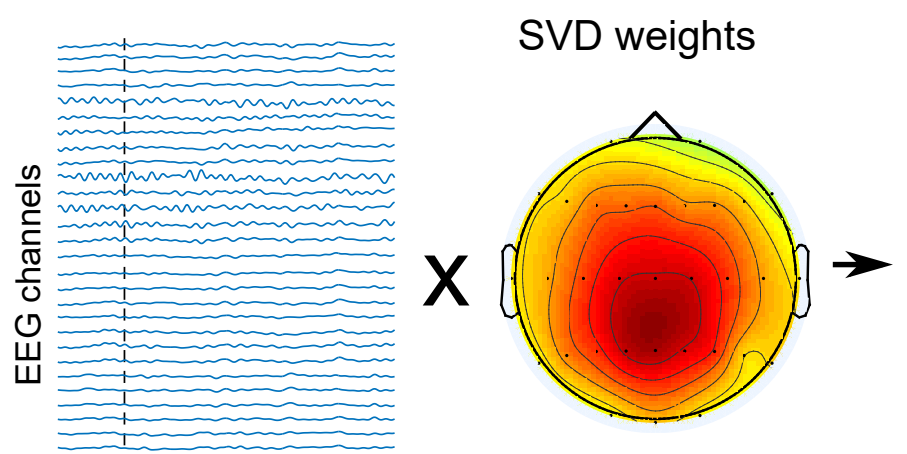

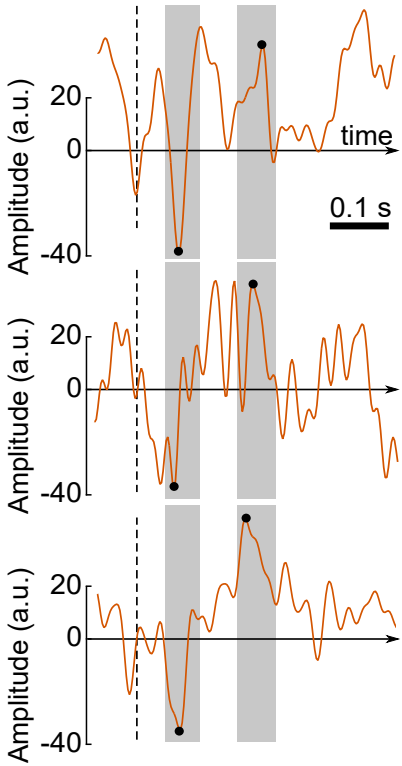

B

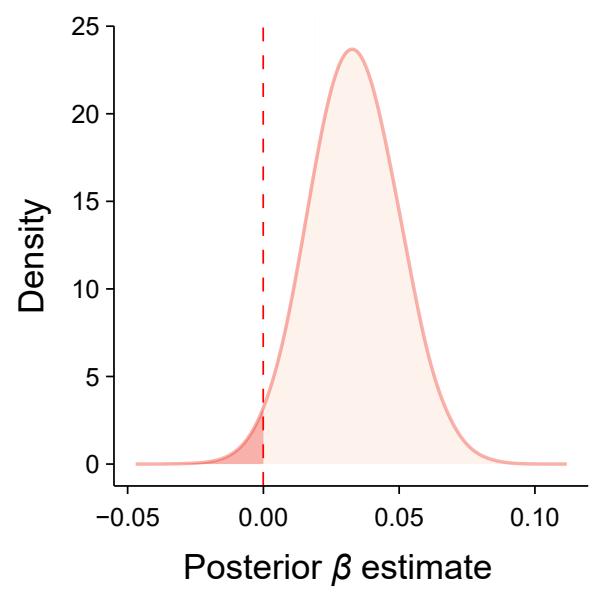

Figure 7. EEG-informed modelling. (A) The schematic diagram of extracting single-trial ERP components. 32-channel EEG signals from a single trial were multiplied by the weights of the first SVD component, calculated from the grand-averaged ERP. Next, the N100 and P300 components in that trial were identified by searching for the peak amplitude in a time of 60-164 ms for the N100 component, and 272-376 ms for the P300 component, respectively. ERP marks in three representative trials were illustrated in the right column of the panel. The ratio between N100-P300 peak amplitude difference and N100-P300 peak latency difference was calculated as a single-trial regressor for modelling. (B) Posterior estimates of the coefficient between the EEG-informed single-trial regressor (i.e., the rising slope of N100-P300 components) and changes in the accumulation rate. 